\title{
ISOGEOMETRIC SCHWARZ PRECONDITIONERS FOR THE BIHARMONIC PROBLEM*
}

\author{
D. $\mathrm{CHO}^{\dagger}$, L. F. PAVARINO ${ }^{\ddagger}$, AND S. SCACCHI ${ }^{\S}$
}

\begin{abstract}
A scalable overlapping Schwarz preconditioner for the biharmonic Dirichlet problem discretized by isogeometric analysis is constructed, and its convergence rate is analyzed. The proposed preconditioner is based on solving local biharmonic problems on overlapping subdomains that form a partition of the CAD domain of the problem and on solving an additional coarse biharmonic problem associated with the subdomain coarse mesh. An $h$-analysis of the preconditioner shows an optimal convergence rate bound that is scalable in the number of subdomains and is cubic in the ratio between subdomain and overlap sizes. Numerical results in $2 \mathrm{D}$ and $3 \mathrm{D}$ confirm this analysis and also illustrate the good convergence properties of the preconditioner with respect to the isogeometric polynomial degree $p$ and regularity $k$.
\end{abstract}

Key words. domain decomposition methods, overlapping Schwarz, biharmonic problem, scalable preconditioners, isogeometric analysis, finite elements, B-splines, NURBS

AMS subject classifications. $65 \mathrm{~N} 55,65 \mathrm{~N} 30,65 \mathrm{~F} 10$

1. Introduction. In the present paper, we construct and analyze overlapping additive Schwarz (OAS) preconditioners for the isogeometric discretization of the following biharmonic problem (see [15, Chapter 5.9]) arising, e.g., in the theory of thin elastic plates, in fluid dynamics when the steady Stokes flow is written in terms of a stream function, or in PDEconstrained optimization (see $[33,39])$ :

$$
\Delta^{2} u=f \quad \text { in } \Omega, \quad u=\frac{\partial u}{\partial n}=0 \quad \text { on } \partial \Omega,
$$

where $\Omega \subset \mathbb{R}^{d}$ is a bounded and connected CAD (computer-aided design) domain.

Typical conforming finite elements for the biharmonic problem need to be $\mathrm{H}^{2}$-conforming, and therefore they require $C^{1}$-continuous piecewise polynomial basis functions leading to complex elements that are more difficult to implement than $H^{1}$-conforming finite elements. The nonconforming finite element alternatives that have been proposed for the biharmonic problem are also known to be quite complex to construct and implement efficiently; see, e.g., [15]. Isogeometric analysis (IGA), introduced more than a decade ago by Hughes et al. [30, 21], employs spline basis functions of degree $p$ and regularity up to $C^{p-1}$, and therefore is well suited for approximating higher-order PDEs such as the biharmonic problem; see, e.g., [40]. The biharmonic equation can also be written in mixed formulation; see, e.g., $[19,34]$ and the references therein. In this paper we confine ourselves to the biharmonic primal formulation.

The novelty of this paper is the extension of overlapping Schwarz preconditioners to the isogeometric discretization of the biharmonic equation. Our two-level OAS preconditioner is

*Received August 14, 2017. Accepted March 6, 2018. Published online on May 2, 2018. Recommended by Axel Klawonn. The work of D. Cho was partially supported by Basic Science Research Program through the National Research Foundation of Korea (NRF) funded by the Ministry of Science, ICT \& Future Planning (2015R1A1A1A05001109). The work of L. F. Pavarino and S. Scacci was partially supported by the Istituto Nazionale di Alta Matematica (INDAM-GNCS).

$\dagger$ Department of Mathematics, Dongguk University, Pil-dong 3-ga, Jung-gu, Seoul, 100-715, South Korea (durkbin@dongguk. edu).

${ }^{\ddagger}$ Dipartimento di Matematica, Università di Pavia, Via Ferrata 1, 27100 Pavia, Italy (luca.pavarino@unipv.it).

§Dipartimento di Matematica, Università di Milano, Via Saldini 50, 20133 Milano, Italy (simone.scacchi@unimi.it). 
based on decomposing the computational domain $\Omega$ into overlapping subdomains $\Omega_{i}$ (which are the images of overlapping sub-patches of the reference domain), solving in parallel local biharmonic problems on each $\Omega_{i}$ and a coarse biharmonic problem on a coarse mesh associated with the subdomain partition of $\Omega$. Our theoretical analysis shows that the resulting preconditioned biharmonic operator satisfies a condition number bound that is scalable in the number of subdomains and is dominated by a $\left(1+\frac{H^{3}}{\gamma^{3}}\right)$-term, where $H$ is the characteristic subdomain size and $\gamma$ is an overlap parameter related to the size of the overlapping region between subdomains. This cubic bound has also been obtained for discontinuous Galerkin finite element discretizations of the biharmonic equation; see [16, 17, 27]. Our theoretical result is confirmed by several numerical experiments on parametric and deformed domains defined by Non-Uniform Rational B-Splines (NURBS) parametrizations, showing additionally a good performance of the biharmonic OAS preconditioner with respect to the spline polynomial degree, regularity, and domain deformation.

Previous works on overlapping domain decomposition for isogeometric discretizations have focused on second-order elliptic problems such as scalar problems with variable coefficients [6, 9], elasticity problems, and second-order saddle-point problems such as the mixed formulation of almost incompressible elasticity and Stokes systems [6, 8]. Other works on IGA preconditioners have focused on nonoverlapping preconditioners of FETI-type for scalar problems [32] and Stokes systems [36], BDDC [7] and BDDC deluxe [11, 12], BPX [18], and multigrid methods [29, 23]. A multipatch isogeometric discretization for the biharmonic equation has been presented in [31]. A discontinuous Galerkin isogeometric discretization of the biharmonic equation has been studied in [35].

Before the introduction of isogeometric analysis, previous domain decomposition methods for the biharmonic equation have considered finite element discretizations [42, 43, 25], discontinuous Galerkin discretizations [16, 17, 27], boundary integrals with a discrete wavelet transform [2], and multilevel radial basis function [1].

This paper is organized as follows. A brief review of B-splines and the basics of NURBS is given in Section 2 together with the isogeometric discretization of the biharmonic model problem. In Section 3, we introduce the two-level overlapping Schwarz preconditioner, while Section 4 presents a condition number bound for the preconditioned operator. Section 5 concludes the paper with several numerical results in 2D and 3D.

2. Isogeometric analysis discretization. We first introduce a compact notation that will be used throughout the rest of the paper. Given two real numbers $a, b$ we write $a \lesssim b$, when $a \leq C b$ for a generic constant $C$ independent of the knot vectors (defined below), and we write $a \approx b$ when $a \lesssim b$ and $b \lesssim a$.

2.1. B-splines notations and spaces. We consider B-splines piecewise polynomial curves in the plane defined as linear combinations of B-spline basis functions. A knot vector is a set of non-decreasing real numbers representing coordinates in the parametric space of the curve

$$
\left\{\xi_{1}=0, \ldots, \xi_{n+p+1}=1\right\}
$$

where $p$ is the polynomial degree of the B-spline and $n$ is the number of basis functions (and control points) describing it. The interval $\left(\xi_{1}, \xi_{n+p+1}\right)$ is called a patch. A knot vector is said to be uniform if its knots are uniformly spaced and non-uniform otherwise. The maximum allowed knot multiplicity is $p+1$; a knot vector is said to be open if its first and last knots have multiplicity $p+1$. In the following, we always employ open knot vectors. Basis functions formed from open knot vectors are interpolatory at the ends of the parametric interval $\widehat{I}:=(0,1)$ but in general are not interpolatory at interior knots. 
Given a knot vector, univariate B-spline basis functions of any degree are defined recursively starting from $p=0$ (piecewise constants); see [37]. The general basis function $N_{i}^{p}$ of degree $p$ has support

$$
\Theta_{i}:=\operatorname{supp}\left(N_{i}^{p}\right)=\left(\xi_{i}, \xi_{i+p+1}\right), \quad i=1,2, \ldots, n .
$$

The functions $N_{i}^{p}$ constitute a partition of unity as shown in [37]. B-spline basis functions are $C^{p-1}$-continuous if internal knots are not repeated. If a knot has multiplicity $\alpha$, then the basis is $C^{k}$-continuous with $k=p-\alpha$ at that knot. In particular, when a knot has multiplicity $\alpha=p$, the basis is $C^{0}$ and interpolates the control point at that location. In the following, we will assume that for polynomial degree $p \geq 2$, the maximum knot multiplicity is $p-1$ so that all considered functions will be (at least) globally $C^{1}$-continuous. The spline space is defined as

$$
\widehat{\mathcal{S}}_{h}=\operatorname{span}\left\{N_{i}^{p}(\xi), i=1, \ldots, n\right\}
$$

Following [37, Theorem 4.41], we will use dual functionals $\lambda_{j}^{p}$ satisfying

$$
\lambda_{j}^{p}\left(N_{i}^{p}\right)=\delta_{i j}, \quad 1 \leq i, j \leq n,
$$

where $\delta_{i j}$ is the Kronecker delta. We recall the following useful estimate for the functional $\lambda_{j}^{p}$ (see [37, Theorem 4.41] for the proof).

LEMmA 2.1. If $f \in L^{q}\left(\xi_{j}, \xi_{j+p+1}\right)$, with $1 \leq q \leq+\infty$, then

$$
\left|\lambda_{j}^{p}(f)\right| \lesssim\left|\xi_{j+p+1}-\xi_{j}\right|^{-1 / q}\|f\|_{L^{q}\left(\xi_{j}, \xi_{j+p+1}\right)} .
$$

Let $\triangle_{i}^{p}:=\xi_{i+p}-\xi_{i}$, for $1 \leq i \leq n$, and $\triangle_{i}^{p-1}:=\xi_{i+p-1}-\xi_{i}$, for $1 \leq i \leq n+1$. We recall the formula for the derivatives of univariate B-splines of degrees $p$ and $p-1$ (see [37, Theorem 4.16]):

$$
\frac{d}{d \xi} N_{i}^{p-1}=(p-1)\left(\frac{N_{i}^{p-2}}{\triangle_{i}^{p-1}}-\frac{N_{i+1}^{p-2}}{\triangle_{i+1}^{p-1}}\right), \quad \frac{d}{d \xi} N_{i}^{p}=p\left(\frac{N_{i}^{p-1}}{\triangle_{i}^{p}}-\frac{N_{i+1}^{p-1}}{\triangle_{i+1}^{p}}\right)
$$

respectively.

A multi-dimensional B-spline space can be constructed by tensor products. For simplicity, we discuss here the case of a two-dimensional space, the higher-dimensional case being analogous. Let $\widehat{\Omega}:=(0,1) \times(0,1)$ be the two-dimensional parametric space. Consider the knot vectors $\left\{\xi_{1}=0, \ldots, \xi_{n+p+1}=1\right\}$ and $\left\{\eta_{1}=0, \ldots, \eta_{m+q+1}=1\right\}$ and also an $n \times m$ net of control points $\mathbf{C}_{i, j}$ that will be used later. The one-dimensional basis functions $N_{i}^{p}$ and $M_{j}^{q}$ (with $i=1, \ldots, n$ and $j=1, \ldots, m$ ) of degree $p$ and $q$, respectively, are defined by the knot vectors. The bivariate spline basis on $\widehat{\Omega}$ is then defined by a tensor product

$$
B_{i, j}^{p, q}(\xi, \eta)=N_{i}^{p}(\xi) M_{j}^{q}(\eta)
$$

The two knot vectors $\left\{\xi_{1}=0, \ldots, \xi_{n+p+1}=1\right\}$ and $\left\{\eta_{1}=0, \ldots, \eta_{m+q+1}=1\right\}$ generate a mesh of rectangular elements in the parametric space in a natural way. Analogous to (2.1), we define the spline space

$$
\widehat{\mathcal{S}}_{h}=\operatorname{span}\left\{B_{i, j}^{p, q}(\xi, \eta), i=1, \ldots, n, j=1, \ldots, m\right\} .
$$


2.2. Non-Uniform Rational B-Splines (NURBS). Rational B-splines in $\mathbb{R}^{d}$ are the projections onto the $d$-dimensional physical space of polynomial B-splines defined in the $(d+1)$-dimensional homogeneous coordinate space; see, e.g., [26] for a complete discussion. A large variety of geometrical entities can be constructed in this way, for example, all conic sections in physical space can be obtained exactly. To obtain a NURBS curve in $\mathbb{R}^{2}$, we introduce the NURBS basis functions of degree $p$

$$
R_{i}^{p}(\xi)=\frac{N_{i}^{p}(\xi) \omega_{i}}{\sum_{\hat{i}=1}^{n} N_{\hat{i}}^{p}(\xi) \omega_{\hat{i}}}=\frac{N_{i}^{p}(\xi) \omega_{i}}{w(\xi)},
$$

where the denominator $w(\xi)=\sum_{\hat{i}=1}^{n} N_{\hat{i}}^{p}(\xi) \omega_{\hat{i}} \in \widehat{\mathcal{S}}_{h}$ is called the weight function.

The NURBS curve is then defined by

$$
\mathbf{C}(\xi)=\sum_{i=1}^{n} R_{i}^{p}(\xi) \mathbf{C}_{i}
$$

where $\mathbf{C}_{i} \in \mathbb{R}^{2}$ are control points.

Analogously to B-splines, NURBS basis functions on the two-dimensional parametric space $\widehat{\Omega}=(0,1) \times(0,1)$ are defined as

$$
R_{i, j}^{p, q}(\xi, \eta)=\frac{B_{i, j}^{p, q}(\xi, \eta) \omega_{i, j}}{\sum_{\hat{i}=1}^{n} \sum_{\hat{j}=1}^{m} B_{\hat{i}, \hat{j}}^{p, q}(\xi, \eta) \omega_{\hat{i}, \hat{j}}}=\frac{B_{i, j}^{p, q}(\xi, \eta) \omega_{i, j}}{w(\xi, \eta)},
$$

where $\omega_{i, j}=\left(\mathbf{C}_{i, j}^{\omega}\right)_{3}$ and the denominator is the weight function denoted also by $w(\xi, \eta)$ (see [30, Equations (9)-(10)] for more details). The continuity and support of NURBS basis functions are the same as for B-splines. NURBS spaces are obtained as the span of the basis functions (2.4) and NURBS regions are defined in terms of the same basis functions. In particular a single-patch domain $\Omega$ is a NURBS region associated with the $n \times m$ net of control points $\mathbf{C}_{i, j}$, and we introduce the geometrical map $\mathbf{F}: \widehat{\Omega} \rightarrow \Omega$ given by

$$
\mathbf{F}(\xi, \eta)=\sum_{i=1}^{n} \sum_{j=1}^{m} R_{i, j}^{p, q}(\xi, \eta) \mathbf{C}_{i, j} .
$$

As in the isoparametric paradigm, the space of NURBS scalar fields on the domain $\Omega$ is defined, component by component, as the span of the push-forward of the basis functions (2.4)

$$
\mathcal{N}_{h}:=\operatorname{span}\left\{R_{i, j}^{p, q} \circ \mathbf{F}^{-1} \text {, with } i=1, \ldots, n, j=1, \ldots, m\right\} .
$$

The image of the elements in the parametric space are elements in the physical space. The physical mesh on $\Omega$ is therefore

$$
\mathcal{T}_{h}=\left\{\mathbf{F}\left(\left(\xi_{i}, \xi_{i+1}\right) \times\left(\eta_{j}, \eta_{j+1}\right)\right), \text { with } i=1, \ldots, n+p, j=1, \ldots, m+q\right\},
$$

where the empty elements are not considered.

2.3. Isogeometric discretization of the biharmonic problem. We can now introduce the isogeometric approximation of the biharmonic model problem (1.1). In order to obtain spaces with homogeneous Dirichlet boundary conditions, it is sufficient to eliminate the first and last two functions in each coordinate. We therefore introduce the spline space (for instance in two dimensions) living in the parameter space

$$
\widehat{V}=\widehat{\mathcal{S}}_{h} \cap H_{0}^{2}(\widehat{\Omega})=\operatorname{span}\left\{B_{i, j}^{p, q}(\xi, \eta), i=3, \ldots, n-2, j=3, \ldots, m-2\right\},
$$




\section{ETNA}

Kent State University and

Johann Radon Institute (RICAM)

and the NURBS space living in physical space

$$
V=\mathcal{N}_{h} \cap H_{0}^{2}(\Omega)=\operatorname{span}\left\{R_{i, j}^{p, q} \circ \mathbf{F}^{-1}, \text { with } i=3, \ldots, n-2, j=3, \ldots, m-2\right\} .
$$

The three-dimensional case is analogous and not discussed here.

The discrete formulation of the model problem (1.1) is then: find $u \in V$ such that

$$
a(u, v)=\int_{\Omega} f v d x \quad \forall v \in V
$$

with the bilinear form $a(u, v)=\int_{\Omega} \Delta u \Delta v d x$.

Throughout the paper we consider homogeneous boundary conditions only for simplicity. Different kind of boundary conditions can be treated in a similar fashion. For instance, (i) when dealing with mixed Dirichlet and Neumann boundary conditions, one determines the knot spans in the parametric space $\widehat{\Omega}$ corresponding to $\Gamma_{D}$ and $\Gamma_{N}$ and then identifies the nonvanishing NURBS that are involved, and (ii) in case of inhomogeneous Dirichlet boundary conditions, we can either interpolate the boundary condition in terms of the basis functions or use quasi-interpolation operators that project the boundary condition into the NURBS space (see [20] for IGA discretizations with inhomogeneous Dirichlet boundary conditions).

3. Overlapping additive Schwarz preconditioners. In this section, we construct an isogeometric overlapping additive Schwarz (OAS) preconditioner for the iterative solution of the discrete problem (2.5). For a general introduction to overlapping Schwarz methods, we refer to, e.g., $[38,41]$.

3.1. Local and coarse subspaces. We start by describing the subdomain decomposition in 1D and then extend it to the higher-dimensional case. The decomposition is first built in the parametric space and then easily extended to the NURBS space in the physical domain. We select from the full set of knots $\left\{\xi_{1}=0, \ldots, \xi_{n+p+1}=1\right\}$ a subset $\left\{\xi_{i_{k}}, k=1, \ldots, N+1\right\}$ of non-repeated interface knots with $\xi_{i_{1}}=0, \xi_{i_{N+1}}=1$. This subset of interface knots defines a decomposition of the closure of the reference interval

$$
\overline{(\widehat{I})}=[0,1]=\overline{\left(\bigcup_{k=1, \ldots, N} \widehat{I}_{k}\right)}, \quad \text { with } \widehat{I}_{k}=\left(\xi_{i_{k}}, \xi_{i_{k+1}}\right),
$$

into $N$ sub-intervals $\widehat{I}_{k}$, for $k=1, \ldots, N$, which we assume to have a similar characteristic diameter $H \approx H_{k}=\operatorname{diam}\left(\widehat{I}_{k}\right)$. The interface knots are thus given by $\xi_{i_{k}}$ for $k=2, \ldots, N$. For each of the interface knots $\xi_{i_{k}}$, we choose an index $3 \leq s_{k} \leq n-2$, strictly increasing in $k$ and satisfying $s_{k}<i_{k}<s_{k}+p+1$, so that the support of the basis function $N_{s_{k}}^{p}$ intersects both $\widehat{I}_{k-1}$ and $\widehat{I}_{k}$. Note that at least one such $s_{k}$ exists; if it is not unique, then any choice can be taken.

An overlapping decomposition of $\widehat{I}$ can then be defined as follows. Let $r \in \mathbb{N}$ be an integer, called overlap index, counting the basis functions shared by adjacent subdomains defined as

$$
\widehat{V}_{k}=\operatorname{span}\left\{N_{j}^{p}(\xi), s_{k}-r \leq j \leq s_{k+1}+r\right\}, \quad k=1,2, \ldots, N,
$$

with the exception that $3 \leq j \leq s_{2}+r$ for the space $\widehat{V}_{1}$ and $s_{N}-r \leq j \leq n-2$ for the space $\widehat{V}_{N}$ (an example is shown in Figure 3.1). These subspaces form an overlapping decomposition of the spline space $\widehat{V}$. For $r=0$ we have the minimal overlap consisting of just one common basis function between adjacent subspaces. We also define the overlap parameter

$$
\gamma=h(2 r+2)
$$




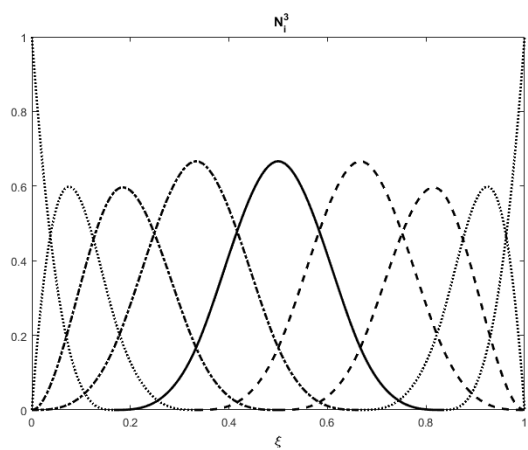

(a) $r=0$

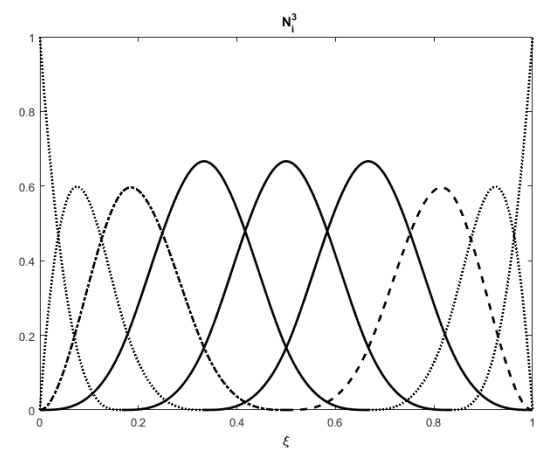

(b) $r=1$

FIG. 3.1. Cubic basis functions associated with $\boldsymbol{\xi}=\{0,0,0,0,1 / 6,1 / 3,1 / 2,2 / 3,5 / 6,1,1,1,1\}$. For various $r, \widehat{V}_{1}$ is the span of basis functions drawn with dash-dotted and solid lines, and $\widehat{V}_{2}$ is the span of basis functions drawn with solid and dashed lines in two subdomains $\widehat{I}_{1}=(0,1 / 2)$ and $\widehat{I}_{2}=(1 / 2,1)$ of $\widehat{I}$. In particular, the basis functions in common are those drawn with a solid line.

which is related to the width $\delta$ of the overlapping region by the following bounds

$$
\gamma=h(2 r+2) \leq \delta \leq h(2 r+p+1) \leq \frac{p+1}{2} \gamma,
$$

where $2 r+1$ represents the number of basis functions in common (in the univariate case) among "adjacent" local subspaces. The extended subdomains $\widehat{I}_{k}^{\prime}$ are defined by

$$
\widehat{I}_{k}^{\prime}=\bigcup_{N_{j}^{p} \in \widehat{V}_{k}} \operatorname{supp}\left(N_{j}^{p}\right)=\left(\xi_{s_{k}-r}, \xi_{s_{k+1}+r+p+1}\right)
$$

with the analogous exception for $\widehat{I}_{1}^{\prime}, \widehat{I}_{N}^{\prime}$, and the further extended subdomains $\widehat{I}_{k}^{\prime \prime}$ by

$$
\widehat{I}_{k}^{\prime \prime}=\bigcup_{\operatorname{supp}\left(N_{j}^{p}\right) \cap \widehat{I}_{k}^{\prime} \neq \emptyset} \operatorname{supp}\left(N_{j}^{p}\right) .
$$

In order to define a coarse space, we introduce an open coarse knot vector

$$
\boldsymbol{\xi}_{0}=\left\{\xi_{1}^{0}=0, \ldots, \xi_{N_{c}+p+1}^{0}=1\right\}
$$

corresponding to a coarse mesh determined by the subdomains $\widehat{I}_{k}$,

$$
\boldsymbol{\xi}_{0}=\left\{\xi_{1}, \xi_{2}, \ldots, \xi_{p}, \xi_{i_{1}}, \xi_{i_{2}}, \xi_{i_{3}}, \ldots, \xi_{i_{N-1}}, \xi_{i_{N}}, \xi_{i_{N}+1}, \xi_{i_{N}+2} \ldots, \xi_{i_{N}+p+1}\right\}
$$

such that the distance between adjacent distinct knots is of order $H$,

$$
\xi_{1}=\cdots=\xi_{p}=\xi_{i_{1}}=0 \quad \text { and } \quad \xi_{i_{N}+1}=\xi_{i_{N}+2}=\cdots=\xi_{i_{N}+p+1}=1
$$

and the associated coarse spline space

$$
\widehat{V}_{0}:=\widehat{\mathcal{S}}_{H}=\operatorname{span}\left\{N_{i}^{0, p}(\xi), i=3, \ldots, N_{c}-2\right\}
$$

has the same degree $p$ of $\widehat{\mathcal{S}}_{h}$ and is thus a subspace of $\widehat{\mathcal{S}}_{h}$. 
In the general higher-dimensional case, we just proceed by tensor products. For example in 2D, we define subdomains, overlapping subdomains, and extended supports by

$$
\begin{aligned}
& \widehat{I}_{k}=\left(\xi_{i_{k}}, \xi_{i_{k+1}}\right), \quad \widehat{I}_{l}=\left(\eta_{j_{l}}, \eta_{j_{l+1}}\right), \quad \widehat{\Omega}_{k l}=\widehat{I}_{k} \times \widehat{I}_{l}, \\
& \widehat{\Omega}_{k l}^{\prime}=\widehat{I}_{k}^{\prime} \times \widehat{I}_{l}^{\prime}, \quad \widehat{\Omega}_{k l}^{\prime \prime}=\widehat{I}_{k}^{\prime \prime} \times \widehat{I}_{l}^{\prime \prime}, \quad 1 \leq k \leq N, 1 \leq l \leq M .
\end{aligned}
$$

Moreover, we take the indexes $\left\{s_{k}\right\}_{k=2}^{N}$ associated to the knots $\left\{\xi_{i_{k}}\right\}_{k=2}^{N}$ and the analogous indexes $\left\{\bar{s}_{l}\right\}_{l=2}^{M}$ associated to the knots $\left\{\eta_{j_{l}}\right\}_{l=2}^{M}$. The local and coarse subspaces are then defined by

$$
\begin{aligned}
\widehat{V}_{k l} & =\operatorname{span}\left\{B_{i, j}^{p, q}(\xi, \eta), s_{k}-r \leq i \leq s_{k+1}+r, \bar{s}_{l}-r \leq j \leq \bar{s}_{l+1}+r\right\}, \\
\widehat{V}_{0} & =\operatorname{span}\left\{\stackrel{\circ}{B}_{i, j}^{p, q}: \stackrel{\circ}{B}_{i, j}^{p, q}(\xi, \eta):=N_{i}^{0, p}(\xi) M_{j}^{0, q}(\eta), i=3, \ldots, N_{c}-2, j=3, \ldots, M_{c}-2\right\},
\end{aligned}
$$

with the usual modification for boundary subdomains and where $\stackrel{\circ}{B}_{i, j}^{p, q}$ are the coarse basis functions.

It is easy to extend the previous decomposition to the NURBS space $V$ in the physical domain. Therefore the local subspaces and the coarse space are, up to the usual modification for the boundary subdomains,

$$
\begin{aligned}
V_{k l} & =\operatorname{span}\left\{R_{i, j}^{p, q} \circ \mathbf{F}^{-1}, s_{k}-r \leq i \leq s_{k+1}+r, \bar{s}_{l}-r \leq j \leq \bar{s}_{l+1}+r\right\}, \\
V_{0} & =\operatorname{span}\left\{\stackrel{\circ}{R}_{i, j}^{p, q} \circ \mathbf{F}^{-1}:=\left(\stackrel{\circ}{B}_{i, j}^{p, q} / w\right) \circ \mathbf{F}^{-1}, i=3, \ldots, N_{c}-2, j=3, \ldots, M_{c}-2\right\},
\end{aligned}
$$

where we recall that $w$ is the weight function; see (2.4). The subdomains in the physical space are defined as the image of the subdomains in the parameter space with respect to the mapping $\mathbf{F}$

$$
\Omega_{k l}=\mathbf{F}\left(\widehat{\Omega}_{k l}\right), \quad \Omega_{k l}^{\prime}=\mathbf{F}\left(\widehat{\Omega}_{k l}^{\prime}\right), \quad \Omega_{k l}^{\prime \prime}=\mathbf{F}\left(\widehat{\Omega}_{k l}^{\prime \prime}\right)
$$

The three-dimensional case is handled analogously.

3.2. The two-level overlapping additive Schwarz operator. Given the embedding operators $\mathbf{I}_{k l}: V_{k l} \rightarrow V, k=1, \ldots, N, l=1, \ldots, M$, and $\mathbf{I}_{0}: V_{0} \rightarrow V$, we define the projections $\widetilde{\mathbf{T}}_{k l}: V \rightarrow V_{k l}$ and $\widetilde{\mathbf{T}}_{0}: V \rightarrow V_{0}$ by

$$
a\left(\widetilde{\mathbf{T}}_{k l} u, v\right)=a\left(u, \mathbf{I}_{k l} v\right) \quad \forall v \in V_{k l}, \quad a\left(\widetilde{\mathbf{T}}_{0} u, v\right)=a\left(u, \mathbf{I}_{0} v\right) \quad \forall v \in V_{0},
$$

and $\mathbf{T}_{k l}=\mathbf{I}_{k l} \widetilde{\mathbf{T}}_{k l}, \mathbf{T}_{0}=\mathbf{I}_{0} \widetilde{\mathbf{T}}_{0}$. The two-level overlapping additive Schwarz (OAS) operator is then

$$
\mathbf{T}_{O A S}:=\mathbf{T}_{0}+\sum_{k=1}^{N} \sum_{l=1}^{M} \mathbf{T}_{k l}
$$

and its matrix form is $\mathbf{T}_{O A S}=\mathbf{B}_{O A S} \mathcal{A}$, where $\mathcal{A}$ is the stiffness matrix and $\mathbf{B}_{O A S}$ is the additive Schwarz preconditioner

$$
\mathbf{B}_{O A S}=R_{0}^{T} A_{0}^{-1} R_{0}+\sum_{k=1}^{N} \sum_{l=1}^{M} R_{k l}^{T} A_{k l}^{-1} R_{k l} .
$$

Here $R_{k l}$ are restriction matrices with 0,1 -entries returning the coefficients of the basis functions belonging to the local spaces $V_{k l}, A_{k l}$ are the local stiffness matrices restricted 
to the subspace $V_{k l}, R_{0}^{T}$ is the coarse-to-fine interpolation matrix, and $A_{0}$ is the coarse stiffness matrix associated with the coarse space $V_{0}$. We also remark that $R_{0}^{T}$ and $R_{k l}^{T}$ are the matrix representations of the operators $\mathbf{I}_{0}$ and $\mathbf{I}_{k l}$, respectively. The use of the $\mathbf{B}_{O A S}$ preconditioner (3.7) for the iterative solution of the discrete system $\mathcal{A} \mathbf{u}=\mathbf{f}$ can also be regarded as replacing it with the preconditioned system

$$
\mathbf{T}_{O A S} \mathbf{u}=\mathbf{g}
$$

where $\mathbf{g}=\mathbf{B}_{O A S} \mathbf{f}$, which can be accelerated by a Krylov subspace method. In the next sections, we will prove a convergence rate bound for the condition number of $\mathbf{T}_{O A S}$.

4. A condition number estimate. In this section, we derive a theoretical estimate for the condition number of the overlapping Schwarz-preconditioned operator. To prove this estimate, we need the following two assumptions on the mesh and subdivision. The first one is a standard assumption also found in the finite element literature, see [41], while the second one allows us to focus on the case of main interest in applications, i.e., when the overlap region is not excessive with respect to the mesh size:

a) the parametric mesh in each extended subdomain $\widehat{\Omega}_{k l}^{\prime \prime}$ is quasi-uniform, i.e., there exists a real number $h=h\left(\widehat{\Omega}_{k l}^{\prime \prime}\right)$ such that all elements $E$ in $\widehat{\Omega}_{k l}^{\prime \prime}$ have a diameter which is equivalent to $h$ up to a constant that is fixed and which is the same for all subdomains;

b) the overlap index $r$ is bounded from above by a fixed constant.

The following theorem is the main theoretical contribution of the present work.

THEOREM 4.1. The condition number of the two-level additive Schwarz preconditioned operator $\mathbf{T}_{O A S}$, defined in (3.6) for the isogeometric biharmonic operator, is bounded by

$$
\kappa_{2}\left(\mathbf{T}_{O A S}\right) \leq C\left(1+\frac{H^{3}}{\gamma^{3}}\right)
$$

where $\gamma=h(2 r+2)$ is the overlap parameter defined in (3.2) and $C$ is a constant independent of $h, H, N, \gamma($ but not of $p, k$ ).

Proof. The proof follows the same steps as those of [6, Theorem 3.1], namely, the general abstract Schwarz theory (see, e.g., [41, Chapter 2]) based on verifying three assumptions known as stable decomposition ([41, Assumption 2.2]), strengthened Cauchy-Schwarz inequality ([41, Assumption 2.3]), and local stability ([41, Assumption 2.4]) that provide an upper and lower bounds on the extreme eigenvalues of $\mathbf{T}_{O A S}$; see [41, Theorem 2.7].

i) Since we use exact local solvers, the local stability assumption holds true with a unit constant.

ii) By using a standard coloring argument (see [41, Chapter 2.5.1]), we obtain that the strengthened Cauchy-Schwarz inequality holds with a constant bounded from above by the number of colors. In the simple case where the nonoverlapping subdomains $\Omega_{i j}$ form a structured Cartesian decomposition of the original domain and the associated overlapping subdomains have a not too large overlap, this constant is 4 in 2D and 8 in 3D.

iii) It remains to prove a stable decomposition ([41, Assumption 2.2]) that will be addressed in the following Sections 4.1 and 4.2 (see Proposition 4.2 for details).

REMARK 4.2. As long as the geometrical map $\mathbf{F}$ is well-behaved in NURBS-based isogeometric methods, an efficient preconditioner on the parametric space can be used as the one for the problems on the physical domain (see e.g. [6, Section 4] for details), which will be demonstrated in various numerical examples in Section 5. Therefore, in the paper we will focus only on the stable splitting for the spline space in the parameter domain.

4.1. Stable decomposition in one dimension. In order to prove the stable decomposition result needed in the proof of Theorem 4.1, we start with some preliminary results for the 
univariate case on which the subsequent analysis will be based. Any $z \in \widehat{V}$ can be represented as a linear combination of $\left\{N_{i}^{p}\right\}_{i=3}^{n-2}$, i.e., there exist constants $c_{j}, 3 \leq j \leq n-2$, such that

$$
z=\sum_{j=3}^{n-2} c_{j} N_{j}^{p}
$$

We first focus on the case of two subdomains, where the difficulties involved in our analysis are already present and then extend the analysis to the general case. As in Section 3.1, we denote the two subdomains by $\widehat{I}_{1}=\left(0, \xi_{i_{2}}\right), \widehat{I}_{2}=\left(\xi_{i_{2}}, 1\right)$. The closure of $\widehat{I}$ is given by $[0,1]$, which is the union of the closures of $\widehat{I}_{1}$ and $\widehat{I}_{2}$; see (3.1). The two associated local spaces are

$$
\widehat{V}_{1}=\operatorname{span}\left\{N_{i}^{p}(\xi), 3 \leq i \leq s_{2}+r\right\}, \quad \widehat{V}_{2}=\operatorname{span}\left\{N_{i}^{p}(\xi), s_{2}-r \leq i \leq n-2\right\},
$$

with $r \geq 0$ and $s_{2}<i_{2}<s_{2}+p+1$. We can write $z$ as in (4.1) and introduce two interpolation operators $\widehat{\Pi}^{k}: \widehat{V} \rightarrow \widehat{V}_{k}, k=1,2$, defined as

$$
z=\sum_{j=3}^{n-2} c_{j} N_{j}^{p}=\widehat{\Pi}^{1} z+\widehat{\Pi}^{2} z \in \widehat{V}_{1}+\widehat{V}_{2},
$$

where

$$
\begin{array}{ll}
\widehat{\Pi}^{1} z=\sum_{j=3}^{s_{2}-r-1} c_{j} N_{j}^{p}+\sum_{j=s_{2}-r}^{s_{2}+r} \frac{\left(s_{2}+r+1\right)-j}{2 r+2} c_{j} N_{j}^{p} \quad \in \widehat{V}_{1}, \\
\widehat{\Pi}^{2} z=\sum_{j=s_{2}-r}^{s_{2}+r} \frac{\left(r-s_{2}+1\right)+j}{2 r+2} c_{j} N_{j}^{p}+\sum_{j=s_{2}+r+1}^{n-2} c_{j} N_{j}^{p} \in \widehat{V}_{2} .
\end{array}
$$

We rewrite $\widehat{\Pi}^{1} z=\sum_{j=3}^{s_{2}+r} \bar{c}_{j} N_{j}^{p}$ with coefficients

$$
\bar{c}_{j}=c_{j} d_{j}, \quad \text { where } \quad d_{j}=\left\{\begin{array}{cl}
1 & \text { if } 3 \leq j \leq s_{2}-r-1, \\
\frac{\left(s_{2}+r+1\right)-j}{2 r+2} & \text { if } s_{2}-r \leq j \leq s_{2}+r
\end{array}\right.
$$

so that $z=\widehat{\Pi}^{1} z+\widehat{\Pi}^{2} z$ holds. Also, by definition and the derivative formula (2.3), we have

$$
\frac{d}{d \xi}\left(\widehat{\Pi}^{1} z\right)=\sum_{j=3}^{s_{2}+r} \bar{c}_{j} \frac{d}{d \xi} N_{j}^{p}=p \sum_{j=3}^{s_{2}+r+1}\left(\bar{c}_{j}-\bar{c}_{j-1}\right) \frac{N_{j}^{p-1}}{\triangle_{j}^{p}},
$$

and

$$
\begin{aligned}
\frac{d^{2}}{d \xi^{2}}\left(\widehat{\Pi}^{1} z\right) & =p \sum_{j=3}^{s_{2}+r+1} \frac{\bar{c}_{j}-\bar{c}_{j-1}}{\triangle_{j}^{p}} \frac{d}{d \xi} N_{j}^{p-1} \\
& =p(p-1) \sum_{j=3}^{s_{2}+r+2}\left(\frac{\bar{c}_{j}-\bar{c}_{j-1}}{\triangle_{j}^{p}}-\frac{\bar{c}_{j-1}-\bar{c}_{j-2}}{\triangle_{j-1}^{p}}\right) \frac{N_{j}^{p-2}}{\triangle_{j}^{p-1}},
\end{aligned}
$$

where we adopted the convention that $\bar{c}_{1}=\bar{c}_{2}=\bar{c}_{s_{2}+r+1}=\bar{c}_{s_{2}+r+2}=0$. From (4.2), $\frac{d^{2}}{d \xi^{2}}\left(\widehat{\Pi}^{1} z\right)(\xi)$ can be represented as a sum of four $T_{i}$-terms, $i=1, \ldots, 4$, as follows: for all $\xi$ 
in $\widehat{I}$,

$$
\begin{aligned}
\frac{d^{2}}{d \xi^{2}}\left(\widehat{\Pi}^{1} z\right)(\xi)= & p(p-1) \sum_{j=3}^{s_{2}+r+2}\left[c_{j}\left(d_{j}-d_{j-1}\right)\right] \frac{N_{j}^{p-2}(\xi)}{\triangle_{j}^{p} \triangle_{j}^{p-1}} \\
& +p(p-1) \sum_{j=3}^{s_{2}+r+2}\left[d_{j-1}\left(c_{j}-c_{j-1}\right)\right] \frac{N_{j}^{p-2}(\xi)}{\triangle_{j}^{p-1} \triangle_{j}^{p}} \\
& +p(p-1) \sum_{j=3}^{s_{2}+r+2}-\left[c_{j-1}\left(d_{j-1}-d_{j-2}\right)\right] \frac{N_{j}^{p-2}(\xi)}{\triangle_{j-1}^{p} \triangle_{j}^{p-1}} \\
& +p(p-1) \sum_{j=3}^{s_{2}+r+2}-\left[d_{j-2}\left(c_{j-1}-c_{j-2}\right)\right] \frac{N_{j}^{p-2}(\xi)}{\triangle_{j-1}^{p} \triangle_{j}^{p-1}} \\
:= & T_{1}(\xi)+T_{2}(\xi)+T_{3}(\xi)+T_{4}(\xi) .
\end{aligned}
$$

Next we prove some estimates on the norms of these $T_{i}$-terms that are needed in order to prove the stable decomposition in one dimension.

LEMMA 4.1. It holds that

$$
\begin{aligned}
\left\|T_{1}(\xi)\right\|_{L^{2}\left(\widehat{I}_{1}^{\prime}\right)}^{2} & \lesssim \frac{H^{3}}{\gamma^{3}}\left\|\frac{d^{2}}{d \xi^{2}} z\right\|_{L^{2}\left(\widehat{I}_{1}^{\prime \prime}\right)}^{2}+\frac{H}{\gamma^{3}}\left\|\frac{d}{d \xi} z\right\|_{L^{2}\left(\widehat{I}_{1}^{\prime \prime}\right)}^{2}+\frac{1}{H \gamma^{3}}\|z\|_{L^{2}\left(\widehat{I}_{1}^{\prime \prime}\right)}^{2}, \\
\left\|T_{3}(\xi)\right\|_{L^{2}\left(\widehat{I}_{1}^{\prime}\right)}^{2} & \lesssim \frac{H^{3}}{\gamma^{3}}\left\|\frac{d^{2}}{d \xi^{2}} z\right\|_{L^{2}\left(\widehat{I}_{1}^{\prime \prime}\right)}^{2}+\frac{H}{\gamma^{3}}\left\|\frac{d}{d \xi} z\right\|_{L^{2}\left(\widehat{I}_{1}^{\prime \prime}\right)}^{2}+\frac{1}{H \gamma^{3}}\|z\|_{L^{2}\left(\widehat{I}_{1}^{\prime \prime}\right)}^{2}, \\
\left\|T_{2}(\xi)+T_{4}(\xi)\right\|_{L^{2}\left(\widehat{I}_{1}^{\prime}\right)}^{2} & \lesssim\left\|\frac{d^{2}}{d \xi^{2}} z\right\|_{L^{2}\left(\widehat{I}_{1}^{\prime \prime}\right)}^{2} .
\end{aligned}
$$

Proof. First, we compute the coefficients

$$
d_{j}-d_{j-1}=\left\{\begin{array}{cl}
0 & \text { if } 3 \leq j \leq s_{2}-r-1 \\
-\theta & \text { if } j \geq s_{2}-r
\end{array}\right.
$$

with $\theta=\frac{1}{2 r+2}$. Due to assumption a) in Section 4 , we have that $\triangle_{j}^{p-1}$ and $\triangle_{j-1}^{p}$ are all greater than or equal to $h$, hence for all $\xi \in \widehat{I}_{1}^{\prime}$, it holds that

$$
\left|T_{1}(\xi)\right| \lesssim \frac{1}{h^{2}} \sum_{s_{2}-r}^{s_{2}+r+2} \theta\left|c_{j}\right| N_{j}^{p-2} .
$$

Property (2.2) of the dual basis and the definition of $z=\sum_{j=3}^{n-2} c_{j} N_{j}^{p}$ imply that $c_{j}=\lambda_{j}^{p}(z)$, hence using Lemma 2.1 with $q=+\infty$ yields for all $s_{2}-r \leq j \leq s_{2}+r+2$

$$
\left|c_{j}\right|=\left.\left|\lambda_{j}^{p}(z)\right| \lesssim|| z\right|_{L^{\infty}\left(\operatorname{supp}\left(T_{1}\right)\right)}
$$

From (4.3) and the partition of unity property of the spline basis functions, this last bound yields

$$
\left|T_{1}(\xi)\right| \lesssim \frac{\theta}{h^{2}} \max _{s_{2}-r \leq j \leq s_{2}+r+2}\left|c_{j}\right| \lesssim \frac{\theta}{h^{2}}\|z\|_{L^{\infty}\left(\operatorname{supp}\left(T_{1}\right)\right)}
$$


Since the support of $T_{1}$ satisfies

$$
\operatorname{supp}\left(T_{1}\right):=\bigcup_{j=s_{2}-r}^{s_{2}+r+2} \operatorname{supp}\left(N_{j}^{p-2}\right)=\left(\xi_{s_{2}-r}, \xi_{s_{2}+r+p+1}\right),
$$

by squaring both sides in (4.4) and integrating over $\widehat{I}_{1}^{\prime}$, we obtain

$$
\begin{aligned}
\int_{\widehat{I}_{1}^{\prime}}\left|T_{1}(\xi)\right|^{2} \mathrm{~d} \xi & =\int_{\operatorname{supp}\left(T_{1}\right)}\left|T_{1}(\xi)\right|^{2} \mathrm{~d} \xi \lesssim\left|\xi_{s_{2}+r+p+1}-\xi_{s_{2}-r}\right|\left\|T_{1}\right\|_{L^{\infty}\left(\operatorname{supp}\left(T_{1}\right)\right)}^{2} \\
& \lesssim \frac{\theta^{2}}{h^{4}}\left|\xi_{s_{2}+r+p+1}-\xi_{s_{2}-r}\right|\|z\|_{L^{\infty}\left(\operatorname{supp}\left(T_{1}\right)\right)}^{2} .
\end{aligned}
$$

Since by (3.2) the overlap parameter is $\gamma=\frac{h}{\theta}=h(2 r+2)$ and by (3.3), we have

$$
\left|\xi_{s_{2}+r+p+1}-\xi_{s_{2}-r}\right| \lesssim(2 r+p+1) h \lesssim \gamma
$$

so that

$$
\int_{\widehat{I}_{1}^{\prime}}\left|T_{1}(\xi)\right|^{2} \mathrm{~d} \xi \lesssim \gamma^{-3}\|z\|_{L^{\infty}\left(\operatorname{supp}\left(T_{1}\right)\right)}^{2} \lesssim \gamma^{-3}\|z\|_{L^{\infty}\left(\widehat{I}_{1}^{\prime \prime}\right)}^{2} .
$$

A standard scaling argument and the $H^{2} \subset L^{\infty}$ one-dimensional Sobolev embedding applied to the above inequality give us the bound

$$
\int_{\widehat{I}_{1}^{\prime}}\left|T_{1}(\xi)\right|^{2} \mathrm{~d} \xi \lesssim \frac{H^{3}}{\gamma^{3}}\left\|\frac{d^{2}}{d \xi^{2}} z\right\|_{L^{2}\left(\widehat{I}_{1}^{\prime \prime}\right)}^{2}+\frac{H}{\gamma^{3}}\left\|\frac{d}{d \xi} z\right\|_{L^{2}\left(\widehat{I}_{1}^{\prime \prime}\right)}^{2}+\frac{1}{H \gamma^{3}}\|z\|_{L^{2}\left(\widehat{I}_{1}^{\prime \prime}\right)}^{2} .
$$

In a similar way, we derive an analogous bound for the $T_{3}$-term

$$
\int_{\widehat{I}_{1}^{\prime}}\left|T_{3}(\xi)\right|^{2} \mathrm{~d} \xi \lesssim \frac{H^{3}}{\gamma^{3}}\left\|\frac{d^{2}}{d \xi^{2}} z\right\|_{L^{2}\left(\widehat{I}_{1}^{\prime \prime}\right)}^{2}+\frac{H}{\gamma^{3}}\left\|\frac{d}{d \xi} z\right\|_{L^{2}\left(\widehat{I}_{1}^{\prime \prime}\right)}^{2}+\frac{1}{H \gamma^{3}}\|z\|_{L^{2}\left(\widehat{I}_{1}^{\prime \prime}\right)}^{2} .
$$

We now estimate $T_{2}(\xi)+T_{4}(\xi)$. For any general function $\varphi=\sum_{j=3}^{n} \beta_{j} N_{j}^{p-2}$, Lemma 2.1 with the choice $q=2$ gives

$$
\left|\beta_{j}\right|=\left|\lambda_{j}^{p-2}(\varphi)\right| \lesssim h^{-1 / 2}\|\varphi\|_{L^{2}\left(\xi_{j}, \xi_{j+p-1}\right)} .
$$

The derivative formulae (2.3) applied to $z=\sum_{j=3}^{n-2} c_{j} N_{j}^{p}$ yield

$$
\frac{d^{2}}{d \xi^{2}} z=p(p-1) \sum_{j=3}^{n}\left(\frac{c_{j}-c_{j-1}}{\triangle_{j}^{p}}-\frac{c_{j-1}-c_{j-2}}{\triangle_{j-1}^{p}}\right) \frac{N_{j}^{p-2}}{\triangle_{j}^{p-1}},
$$

and from (4.5) we obtain

$$
p(p-1)\left|\left(\frac{c_{j}-c_{j-1}}{\triangle_{j}^{p}}-\frac{c_{j-1}-c_{j-2}}{\triangle_{j-1}^{p}}\right) \frac{1}{\triangle_{j}^{p-1}}\right| \lesssim h^{-1 / 2}\left\|\frac{d^{2}}{d \xi^{2}} z\right\|_{L^{2}\left(\xi_{j}, \xi_{j+p-1}\right)},
$$

(we have adopted the convention $c_{1}=c_{2}=c_{n-1}=c_{n}=0$ ). From the definition of $d_{j}$, the term $T_{2}+T_{4}$ can be rewritten as

$$
\begin{aligned}
T_{2}+T_{4}= & p(p-1) \sum_{j=3}^{s_{2}-r}\left(\frac{c_{j}-c_{j-1}}{\triangle_{j}^{p}}-\frac{c_{j-1}-c_{j-2}}{\triangle_{j-1}^{p}}\right) \frac{N_{j}^{p-2}}{\triangle_{j}^{p-1}} \\
& +p(p-1) \sum_{j=s_{2}-r+1}^{s_{2}+r+2}\left(\frac{d_{j-1}\left(c_{j}-c_{j-1}\right)}{\triangle_{j}^{p}}-\frac{d_{j-2}\left(c_{j-1}-c_{j-2}\right)}{\triangle_{j-1}^{p}}\right) \frac{N_{j}^{p-2}}{\triangle_{j}^{p-1}}
\end{aligned}
$$


By comparing with (4.6), we note that the restriction of $T_{2}+T_{4}$ to $\left(0, \xi_{s_{2}-r+1}\right)$ coincides with $\frac{d^{2}}{d \xi^{2}} z$, hence,

$$
\begin{aligned}
\int_{\widehat{I}_{1}^{\prime}} \mid & T_{2}(\xi)+\left.T_{4}(\xi)\right|^{2} \mathrm{~d} \xi=\int_{\left(0, \xi_{s_{2}-r+1}\right)}\left|T_{2}(\xi)+T_{4}(\xi)\right|^{2} \mathrm{~d} \xi \\
& +\int_{\left(\xi_{s_{2}-r+1}, \xi_{s_{2}+r+p+1}\right)}\left|T_{2}(\xi)+T_{4}(\xi)\right|^{2} \mathrm{~d} \xi \\
=\int_{\left(0, \xi_{s_{2}-r+1}\right)}\left|\frac{d^{2}}{d \xi^{2}} z\right|^{2} \mathrm{~d} \xi & +\int_{\left(\xi_{s_{2}-r+1}, \xi_{s_{2}+r+p+1}\right)}\left|T_{2}(\xi)+T_{4}(\xi)\right|^{2} \mathrm{~d} \xi
\end{aligned}
$$

From the partition of unity property, the bound (4.7), and the definition of $T_{2}$ and $T_{4}$, for all $\xi \in \operatorname{supp}\left(T_{2}+T_{4}\right)$, we have

$$
\begin{aligned}
\left|T_{2}(\xi)+T_{4}(\xi)\right| & \lesssim \max _{3 \leq j \leq s_{2}+r+2} p(p-1)\left|\left(\frac{c_{j}-c_{j-1}}{\triangle_{j}^{p}}-\frac{c_{j-1}-c_{j-2}}{\triangle_{j-1}^{p}}\right) \frac{1}{\triangle_{j}^{p-1}}\right| \\
& \lesssim h^{-1 / 2}\left\|\frac{d^{2}}{d \xi^{2}} z\right\|_{L^{2}\left(0, \xi_{s_{2}+r+p+1}\right)} \cdot
\end{aligned}
$$

By Hölder's inequality, (4.9), and assumptions a), b) from Section 4, and (4.8), we obtain

$$
\begin{aligned}
\int_{\widehat{I}_{1}^{\prime}} \mid & T_{2}(\xi)+\left.T_{4}(\xi)\right|^{2} \mathrm{~d} \xi \\
& \lesssim\left\|\frac{d^{2}}{d \xi^{2}} z\right\|_{L^{2}\left(\widehat{I}_{1}\right)}^{2}+\left|\xi_{s_{2}+r+p+1}-\xi_{s_{2}-r+1}\right|\left\|T_{2}+T_{4}\right\|_{L^{\infty}\left(\xi_{s_{2}-r+1}, \xi_{s_{2}+r+p+1}\right)}^{2} \\
& \lesssim\left\|\frac{d^{2}}{d \xi^{2}} z\right\|_{L^{2}\left(\widehat{I}_{1}\right)}^{2}+h^{-1}\left|\xi_{s_{2}+r+p+1}-\xi_{s_{2}-r+1}\right|\left\|\frac{d^{2}}{d \xi^{2}} z\right\|_{L^{2}\left(0, \xi_{s_{2}+r+p+1}\right)}^{2} \\
& \lesssim\left\|\frac{d^{2}}{d \xi^{2}} z\right\|_{L^{2}\left(\widehat{I}_{1}^{\prime \prime}\right)}^{2} \cdot
\end{aligned}
$$

We are now in a position to show the following stability bounds for the two-subdomain decomposition. $z \in \widehat{V}$

PROPOSITION 4.1. The operators $\widehat{\Pi}^{k}, k=1,2$, satisfy the following bounds for all

$$
\begin{aligned}
\left\|\frac{d^{2}}{d \xi^{2}}\left(\widehat{\Pi}^{k} z\right)\right\|_{L^{2}\left(\widehat{I}_{k}^{\prime}\right)}^{2} & \lesssim\left(1+\frac{H^{3}}{\gamma^{3}}\right)\left\|\frac{d^{2}}{d \xi^{2}} z\right\|_{L^{2}\left(\widehat{I}_{k}^{\prime \prime}\right)}^{2}+\frac{H}{\gamma^{3}}\left\|\frac{d}{d \xi} z\right\|_{L^{2}\left(\widehat{I}_{k}^{\prime \prime}\right)}^{2}+\frac{1}{H \gamma^{3}}\|z\|_{L^{2}\left(\widehat{I}_{k}^{\prime \prime}\right)}^{2} \\
\left\|\widehat{\Pi}^{k} z\right\|_{L^{2}\left(\widehat{I}_{k}^{\prime}\right)} & \lesssim\|z\|_{L^{2}\left(\widehat{I}_{k}^{\prime \prime}\right)}
\end{aligned}
$$

where $\widehat{I}_{k}^{\prime}$ and $\widehat{I}_{k}^{\prime \prime}$ are defined in (3.4) and (3.5), respectively.

Proof. We prove the result only for $\widehat{\Pi}^{1}$ since the result for $\widehat{\Pi}^{2}$ follows from the same argument. The first bound (4.10) follows from Lemma 4.1. To prove the second bound (4.11), consider an element $e=\left(\xi_{m}, \xi_{m+1}\right) \subset \widehat{I}_{1}^{\prime}$ of the mesh. Lemma 2.1 with $q=2$, implies that for all $\xi \in e$, we have

$$
\widehat{\Pi}^{1} z(\xi)=\sum_{j=m-p}^{m} \bar{c}_{j} N_{j}^{p}(\xi) \leq \max _{m-p \leq j \leq m}\left|c_{j}\right| \lesssim h^{-1 / 2}\|z\|_{L^{2}\left(\xi_{m-p}, \xi_{m+p+1}\right)},
$$


since $\left|\bar{c}_{j}\right| \leq\left|c_{j}\right|$ for all values of $j$. Hence,

$$
\int_{e}\left|\widehat{\Pi}^{1} z\right|^{2} \lesssim h^{-1} h\|z\|_{L^{2}\left(\xi_{m-p}, \xi_{m+p+1}\right)}^{2}=\|z\|_{L^{2}\left(\xi_{m-p}, \xi_{m+p+1}\right)}^{2} .
$$

The proof is completed by summing over all elements in the mesh, yielding that $\left\|\widehat{\Pi}^{1} z\right\|_{L^{2}\left(\widehat{I}_{1}^{\prime}\right)} \lesssim\|z\|_{L^{2}\left(\widehat{I}_{1}^{\prime \prime}\right)}$.

These results can be directly extended to the general case with $N$ subdomains. If

$$
z=\sum_{j=3}^{n-2} c_{j} N_{j}^{p}
$$

then

$$
z=\sum_{k=1}^{N} \widehat{\Pi}^{k} z,
$$

with the interpolation operators $\widehat{\Pi}^{k}: \widehat{V} \rightarrow \widehat{V}_{k}$ defined by

$$
\begin{aligned}
\widehat{\Pi}^{k} z= & \sum_{j=s_{k}-r}^{s_{k}+r} \frac{\left(r-s_{k}+1\right)+j}{2 r+2} c_{j} N_{j}^{p}+\sum_{j=s_{k}+r+1}^{s_{k+1}-r-1} c_{j} N_{j}^{p} \\
& +\sum_{j=s_{k+1}-r}^{s_{k+1}+r} \frac{\left(s_{k+1}+r+1\right)-j}{2 r+2} c_{j} N_{j}^{p},
\end{aligned}
$$

(with the usual modification for the two boundary subdomains and where we assume the condition $s_{k}+r+1 \leq s_{k+1}-r-1$ to avoid the overlap of non-adjacent subdomains).

Following the same arguments presented in the two-subdomain case, we obtain the following result. $z \in \widehat{V}$

THEOREM 4.1. The operators $\widehat{\Pi}^{k}, k=1,2, \ldots, N$, satisfy the following bounds for all

$$
\begin{aligned}
\left\|\frac{d^{2}}{d \xi^{2}}\left(\widehat{\Pi}^{k} z\right)\right\|_{L^{2}\left(\widehat{I}_{k}^{\prime}\right)}^{2} & \lesssim\left(1+\frac{H^{3}}{\gamma^{3}}\right)\left\|\frac{d^{2}}{d \xi^{2}} z\right\|_{L^{2}\left(\widehat{I}_{k}^{\prime \prime}\right)}^{2}+\frac{H}{\gamma^{3}}\left\|\frac{d}{d \xi} z\right\|_{L^{2}\left(\widehat{I}_{k}^{\prime \prime}\right)}^{2}+\frac{1}{H \gamma^{3}}\|z\|_{L^{2}\left(\widehat{I}_{k}^{\prime \prime}\right)}^{2}, \\
\left\|\widehat{\Pi}^{k} z\right\|_{L^{2}\left(\widehat{I}_{k}^{\prime}\right)} & \lesssim\|z\|_{L^{2}\left(\widehat{I}_{k}^{\prime \prime}\right)} .
\end{aligned}
$$

4.2. Stable decomposition in two dimensions. We now turn to the two-dimensional case (the three-dimensional case is analogous). By applying standard tensorization arguments, we define the linear operators

$$
\begin{aligned}
& \widehat{\Pi}^{k l}: \widehat{V} \longrightarrow \widehat{V}_{k l}, \\
& \widehat{\Pi}^{k l} v=\widehat{\Pi}_{\boldsymbol{\xi}}^{k} \otimes \widehat{\Pi}_{\boldsymbol{\eta}}^{l}(v) \quad \forall v \in \widehat{V},
\end{aligned}
$$

where $\widehat{\Pi}_{\xi}^{k}$ and $\widehat{\Pi}_{\boldsymbol{\eta}}^{l}$ are one-dimensional operators generated by the knot vectors $\boldsymbol{\xi}$ and $\boldsymbol{\eta}$ in the previous section. Define $\widehat{\Pi}^{0}$ to be the standard spline quasi-interpolant into the space $\widehat{V}_{0}$, which is built using the dual basis functions as detailed in [37, Theorem 12.6]. Given $\widehat{u} \in \widehat{V}$, we define

$$
\widehat{z}=\widehat{u}-\widehat{u}_{0} \in \widehat{V}, \quad \widehat{u}_{0}=\widehat{\Pi}^{0} \widehat{u} \in \widehat{V}_{0} .
$$


From the stability and approximation properties of the quasi-interpolant, see [37, Theorem 12.7], it follows that

$$
\| \widehat{z}||_{L^{2}(\widehat{\Omega})}+H|\widehat{z}|_{H^{1}(\widehat{\Omega})}+H^{2}\left|\widehat{u}_{0}\right|_{H^{2}(\widehat{\Omega})} \lesssim H^{2}|\widehat{u}|_{H^{2}(\widehat{\Omega})} .
$$

Using the tensorized definition of $\widehat{\Pi}^{k l}$ and the splitting property (4.12) of the one-dimensional operators, we obtain

$$
\sum_{k=1}^{N} \sum_{l=1}^{M} \widehat{\Pi}^{k l} \widehat{z}=\sum_{k=1}^{N} \widehat{\Pi}_{\xi}^{k}\left(\sum_{l=1}^{M} \widehat{\Pi}_{\boldsymbol{\eta}}^{l} \widehat{z}\right)=\sum_{k=1}^{N} \widehat{\Pi}_{\xi}^{k} \widehat{z}=\widehat{z}
$$

so that denoting $\widehat{u}_{k l}=\widehat{\Pi}^{k l} \widehat{z}$ we arrive at the splitting

$$
\widehat{u}=\widehat{u}_{0}+\sum_{k=1}^{N} \sum_{l=1}^{M} \widehat{u}_{k l}, \quad \widehat{u}_{0} \in \widehat{V}_{0}, \widehat{u}_{k l} \in \widehat{V}_{k l} .
$$

We can now prove the stability of this splitting.

Proposition 4.2. Given any $\widehat{u} \in \widehat{V}$ decomposed as in (4.17), we have

$$
\left|\widehat{u}_{0}\right|_{H^{2}(\widehat{\Omega})}^{2}+\sum_{k=1}^{N} \sum_{l=1}^{M}\left|\widehat{u}_{k l}\right|_{H^{2}(\widehat{\Omega})}^{2} \lesssim\left(1+\frac{H^{3}}{\gamma^{3}}\right)|\widehat{u}|_{H^{2}(\widehat{\Omega})}^{2} .
$$

Proof. The bound for the coarse term $\widehat{u}_{0}$ follows from (4.16). In order to bound the local terms $\widehat{u}_{k l}, 1 \leq k \leq N$ and $1 \leq l \leq M$, we consider only the $\xi$-derivative since the result for the $\eta$-derivative will follow by the same arguments. From the tensorized definition (4.15) and since $\operatorname{supp}\left(\widehat{u}_{k l}\right)=\widehat{\Omega}_{k l}^{\prime}$, we have

$$
\left\|\frac{\partial^{2}}{\partial \xi^{2}} \widehat{u}_{k l}\right\|_{L^{2}(\widehat{\Omega})}^{2}=\left\|\frac{\partial^{2}}{\partial \xi^{2}}\left(\widehat{\Pi}_{\boldsymbol{\eta}}^{l} \otimes \widehat{\Pi}_{\boldsymbol{\xi}}^{k}\right) \widehat{z}\right\|_{L^{2}\left(\widehat{\Omega}_{k l}^{\prime}\right)}^{2}=\left\|\widehat{\Pi}_{\boldsymbol{\eta}}^{l} \frac{\partial^{2}}{\partial \xi^{2}}\left(\widehat{\Pi}_{\boldsymbol{\xi}}^{k} \widehat{z}\right)\right\|_{L^{2}\left(\widehat{\Omega}_{k l}^{\prime}\right)}^{2} .
$$

From the one-dimensional bound (4.14) in Theorem 4.1 and the definition of $\widehat{\Omega}_{k l}^{\prime}$, we have for any $v \in \widehat{V}$

$$
\left\|\widehat{\Pi}_{\eta}^{l} v\right\|_{L^{2}\left(\widehat{\Omega}_{k l}^{\prime}\right)}^{2}=\int_{\widehat{I}_{k}^{\prime}} \int_{\widehat{I}_{l}^{\prime}}\left|\left(\widehat{\Pi}_{\eta}^{l} v\right)(\xi, \eta)\right|^{2} \mathrm{~d} \eta \mathrm{d} \xi \lesssim \int_{\widehat{I}_{k}^{\prime}} \int_{\widehat{I}_{l}^{\prime \prime}}|v(\xi, \eta)|^{2} \mathrm{~d} \eta \mathrm{d} \xi
$$

From (4.19), (4.18), the tensorized definition (4.15), and the one-dimensional bound (4.13), we obtain

$$
\begin{aligned}
\left\|\frac{\partial^{2}}{\partial \xi^{2}} \widehat{u}_{k l}\right\|_{L^{2}(\widehat{\Omega})}^{2} & \lesssim \int_{\widehat{I}_{k}^{\prime}} \int_{\widehat{I}_{l}^{\prime \prime}}\left|\frac{\partial^{2}}{\partial \xi^{2}}\left(\widehat{\Pi}_{\xi}^{k} \widehat{z}\right)(\xi, \eta)\right|^{2} \mathrm{~d} \eta \mathrm{d} \xi=\int_{\widehat{I}_{l}^{\prime \prime}} \int_{\widehat{I}_{k}^{\prime}}\left|\frac{\partial^{2}}{\partial \xi^{2}}\left(\widehat{\Pi}_{\xi}^{k} \widehat{z}\right)(\xi, \eta)\right|^{2} \mathrm{~d} \xi \mathrm{d} \eta \\
& \lesssim\left(1+\frac{H^{3}}{\gamma^{3}}\right)\left\|\frac{\partial^{2}}{\partial \xi^{2}} \widehat{z}\right\|_{L^{2}\left(\widehat{\Omega}_{k l}^{\prime \prime}\right)}^{2}+\frac{H}{\gamma^{3}}\left\|\frac{\partial}{\partial \xi} \widehat{z}\right\|_{L^{2}\left(\widehat{\Omega}_{k l}^{\prime \prime}\right)}^{2}+\frac{1}{H \gamma^{3}}\|\widehat{z}\|_{L^{2}\left(\widehat{\Omega}_{k l}^{\prime \prime}\right)}^{2}
\end{aligned}
$$

By a standard coloring argument, the number of extended subdomains $\widehat{\Omega}_{k l}^{\prime \prime}$ overlapping at a given point is uniformly bounded, hence (4.20) gives

$$
\sum_{k=1}^{N} \sum_{l=1}^{M}\left\|\frac{\partial^{2}}{\partial \xi^{2}} \widehat{u}_{k l}\right\|_{L^{2}(\widehat{\Omega})}^{2} \lesssim\left(1+\frac{H^{3}}{\gamma^{3}}\right)\left\|\frac{\partial^{2}}{\partial \xi^{2}} \widehat{z}\right\|_{L^{2}(\widehat{\Omega})}^{2}+\frac{H}{\gamma^{3}}\left\|\frac{\partial}{\partial \xi} \widehat{z}\right\|_{L^{2}(\widehat{\Omega})}^{2}+\frac{1}{H \gamma^{3}}\|\widehat{z}\|_{L^{2}(\widehat{\Omega})}^{2},
$$



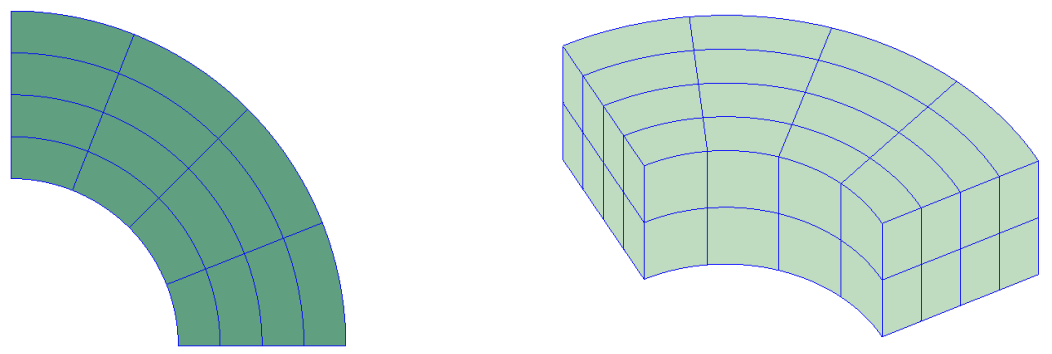

FIG. 5.1. Quarter-ring domain (left) and thick quarter-ring domain (right) used in the numerical tests.

which in turn, using the definition of $\widehat{\Omega}$ and property (4.16), leads to

$$
\sum_{k=1}^{N} \sum_{l=1}^{M}\left\|\frac{\partial^{2}}{\partial \xi^{2}} \widehat{u}_{k l}\right\|_{L^{2}(\widehat{\Omega})}^{2} \lesssim\left(1+\frac{H^{3}}{\gamma^{3}}\right)|\widehat{u}|_{H^{2}(\widehat{\Omega})}^{2}
$$

The stable splitting of Proposition 4.2 together with the steps of the abstract Schwarz theory of Section 4 prove Theorem 4.1 for the case of the spline space in the parameter domain.

5. Numerical results. In this section, we report the results of several numerical tests with our two-level overlapping Schwarz preconditioner OAS(2) (see (3.7)) applied to the isogeometric discretization of the biharmonic model problem (1.1) in two and and three dimensions, using the MATLAB isogeometric library GeoPDEs [22]. The isogeometric discretization parameters are the mesh size $h$, the polynomial degree $p$, and the regularity $k$. The computational domain is decomposed into $N$ overlapping subdomains of characteristic size $H$, and the overlap size is given by the overlap index $r$ described in Section 3. The tests are run on both parametric domains (square, cubic) and deformed domains (quarter-ring and thick-quarter ring, see Figure 5.1). The OAS(2) preconditioner is accelerated by the preconditioned conjugate gradient (PCG), with zero initial guess and as stopping criterion a $10^{-6}$ reduction of the relative residual.

5.1. 2D tests: $\mathrm{OAS}(2)$ scalability in $N$ and optimality in $H / h$. The condition number $\left(\kappa_{2}\right)$ and the iteration counts (it) of the OAS(2) preconditioner are reported in Table 5.1 for the reference square and in Table 5.2 for a quarter-ring physical domain as a function of the number of subdomains $N$ and the mesh size $1 / h$ for fixed $p=2, k=1$ splines parameter and overlap $r=0$ and $r=1$. Additional results for different splines spaces $p=3, k=2$ and $p=3, k=1$ are reported in the Tables 5.3, 5.4, 5.5, 5.6. The results show that the proposed preconditioner is scalable since moving along the diagonal of the tables, the condition number is bounded above by a constant independent of $N$; see Figure 5.2. The results of all tables and figures also confirm the main bound of Theorem 4.1: moving along each table row, the condition numbers exhibit a cubic growth with the increasing ratio $H / h$ (here for a fixed overlap index $r$ we have $H / \gamma=O(H / h)$ ); see Figure 5.3. Moreover, these numerical results imply that the main scalability and quasi-optimality properties of our proposed preconditioner also hold for the deformed geometry of the quarter-ring domain (Table 5.2) since the increasing ill-conditioning of the deformed model on the quarter-ring domain yields worse iteration counts and condition numbers, but the associated plots of Figure 5.2 show that this growth is still confined, and the preconditioner is scalable.

5.2. 2D tests: OAS(2) dependence on $p$ and $k$. We now investigate the performance of the OAS biharmonic preconditioner for an increasing polynomial degree $p=2, \ldots, 12$, 


\section{ETNA}

Kent State University and

Johann Radon Institute (RICAM)

TABLE 5.1

$2 D O A S(2)$ scalability and optimality test on the unit square domain: condition number $\kappa_{2}$ of the preconditioned operator and PCG iteration counts (it) in brackets as a function of the number of subdomains $N$ and mesh size $1 / h$. $p=2, k=1$ B-splines.

OAS(2) prec. with $r=0, p=2, k=1$ B-splines, square domain

\begin{tabular}{c|r|r|r|r|r|r} 
& $1 / h=8$ & $1 / h=16$ & $1 / h=32$ & $1 / h=64$ & $1 / h=128$ & $1 / h=256$ \\
$\kappa_{2}$ (it) & $\kappa_{2}$ (it) & $\kappa_{2}$ (it) & $\kappa_{2}$ (it) & $\kappa_{2}$ (it) & $\kappa_{2}$ (it) \\
\hline $2 \times 2$ & $7.68(6)$ & $36.32(10)$ & $230.23(14)$ & $1.70 \mathrm{e}+3(23)$ & $1.34 \mathrm{e}+4(43)$ & $1.06 \mathrm{e}+5(85)$ \\
$4 \times 4$ & & $9.82(15)$ & $45.82(23)$ & $288.97(43)$ & $2.14 \mathrm{e}+3(81)$ & $1.67 \mathrm{e}+4(162)$ \\
$8 \times 8$ & & & $10.22(23)$ & $44.69(41)$ & $274.61(87)$ & $2.01 \mathrm{e}+3(212)$ \\
$16 \times 16$ & & & & $10.41(25)$ & $43.95(49)$ & $264.53(113)$ \\
$32 \times 32$ & & & & & $10.44(25)$ & $43.97(54)$ \\
$64 \times 64$ & & & & & & $10.45(25)$
\end{tabular}

OAS(2) prec. with $r=1, \quad p=2, k=1$ B-splines, square domain

\begin{tabular}{c|r|r|r|r|r|r} 
& $1 / h=8$ & $1 / h=16$ & $1 / h=32$ & $1 / h=64$ & $1 / h=128$ & $1 / h=256$ \\
$N$ & $\kappa_{2}$ (it) & $\kappa_{2}$ (it) & $\kappa_{2}$ (it) & $\kappa_{2}$ (it) & $\kappa_{2}$ (it) & $\kappa_{2}$ (it) \\
\hline $2 \times 2$ & $3.86(4)$ & $13.53(10)$ & $73.28(13)$ & $503.14(19)$ & $3.85 \mathrm{e}+3(30)$ & $3.04 \mathrm{e}+4(58)$ \\
$4 \times 4$ & & $5.92(15)$ & $17.25(19)$ & $92.31(30)$ & $631.11(56)$ & $4.82 \mathrm{e}+3(109)$ \\
$8 \times 8$ & & & $7.39(21)$ & $17.38(30)$ & $88.92(56)$ & $596.84(127)$ \\
$16 \times 16$ & & & & $7.47(22)$ & $17.52(33)$ & $86.51(70)$ \\
$32 \times 32$ & & & & & $7.49(22)$ & $17.59(35)$ \\
$64 \times 64$ & & & & & & $7.48(22)$
\end{tabular}

TABLE 5.2

$2 D O A S(2)$ scalability and optimality test on the quarter-ring domain: condition number of the preconditioned operator $\left(\kappa_{2}\right)$ and PCG iteration counts (it) in brackets as a function of the number of subdomains $N$ and mesh size $1 / h . p=2, k=1$ NURBS.

\begin{tabular}{c|r|r|r|r|r|r}
\multicolumn{8}{c}{ OAS(2) prec. with $r=0, p=2, k=1$ NURBS, quarter-ring domain } \\
\multicolumn{1}{c|}{$1 / h=8$} & $1 / h=16$ & $1 / h=32$ & $1 / h=64$ & $1 / h=128$ & $1 / h=256$ \\
$N$ & $\kappa_{2}$ (it) & $\kappa_{2}$ (it) & $\kappa_{2}$ (it) & $\kappa_{2}$ (it) & $\kappa_{2}$ (it) & $\kappa_{2}$ (it) \\
\hline $2 \times 2$ & $8.21(11)$ & $38.52(18)$ & $256.35(33)$ & $1.97 \mathrm{e}+3(62)$ & $1.57 \mathrm{e}+4(124)$ & $1.26 \mathrm{e}+5(256)$ \\
$4 \times 4$ & & $22.99(26)$ & $156.85(53)$ & $1.22 \mathrm{e}+3(135)$ & $9.87 \mathrm{e}+3(317)$ & $7.99 \mathrm{e}+4(724)$ \\
$8 \times 8$ & & & $41.21(41)$ & $301.62(99)$ & $2.44 \mathrm{e}+3(268)$ & $2.00 \mathrm{e}+4(758)$ \\
$16 \times 16$ & & & & $55.76(55)$ & $413.02(146)$ & $3.38 \mathrm{e}+3(421)$ \\
$32 \times 32$ & & & & & $68.22(63)$ & $507.10(190)$ \\
$64 \times 64$ & & & & & & $77.87(73)$
\end{tabular}

OAS(2) prec. with $r=1, \quad p=2, k=1$ NURBS, quarter-ring domain

\begin{tabular}{c|r|r|r|r|r|r} 
& $1 / h=8$ & $1 / h=16$ & $1 / h=32$ & $1 / h=64$ & $1 / h=128$ & $1 / h=256$ \\
$N$ & $\kappa_{2}$ (it) & $\kappa_{2}$ (it) & $\kappa_{2}$ (it) & $\kappa_{2}$ (it) & $\kappa_{2}(\mathrm{it})$ & $\kappa_{2}$ (it) \\
\hline $2 \times 2$ & $4.36(9)$ & $14.18(15)$ & $78.96(25)$ & $568.95(43)$ & $4.47 \mathrm{e}+3(84)$ & $3.59 \mathrm{e}+4(171)$ \\
$4 \times 4$ & & $11.58(21)$ & $48.00(34)$ & $349.50(79)$ & $2.79 \mathrm{e}+3(195)$ & $2.26 \mathrm{e}+4(448)$ \\
$8 \times 8$ & & & $20.70(29)$ & $89.31(58)$ & $686.02(151)$ & $5.61 \mathrm{e}+3(414)$ \\
$16 \times 16$ & & & & $27.94(39)$ & $121.83(83)$ & $945.61(230)$ \\
$32 \times 32$ & & & & & $33.93(45)$ & $149.96(103)$ \\
$64 \times 64$ & & & & & & $38.52(52)$
\end{tabular}

while keeping other parameters fixed, $1 / h=32, N=2 \times 2, H / h=16$. Table 5.7 displays the condition number $\kappa_{2}\left(T_{O A S}\right)$ (and iterations in brackets) as a function of $p$ for the maximal regularity $k=p-1$ (left columns) and the minimal regularity $k=1$ (right columns) with different levels of overlap from symmetric minimal $(r=0)$ to symmetric generous $(r=p)$. 


\section{ETNA}

Kent State University and Johann Radon Institute (RICAM)

TABLE 5.3

$2 D O A S(2)$ scalability and optimality test on the unit square domain: condition number of the preconditioned operator $\left(\kappa_{2}\right)$ and PCG iteration counts (it) in brackets as a function of the number of subdomains $N$ and mesh size $1 / h . p=3, k=2$ B-splines.

OAS(2) prec. with $r=0, p=3, k=2$ B-splines, square domain

\begin{tabular}{c|r|r|r|r|r|r} 
& $1 / h=8$ & $1 / h=16$ & $1 / h=32$ & $1 / h=64$ & $1 / h=128$ & $1 / h=256$ \\
$N$ & $\kappa_{2}$ (it) & $\kappa_{2}(\mathrm{it})$ & $\kappa_{2}(\mathrm{it})$ & $\kappa_{2}(\mathrm{it})$ & $\kappa_{2}(\mathrm{it})$ & $\kappa_{2}(\mathrm{it})$ \\
\hline $2 \times 2$ & $5.03(8)$ & $8.10(11)$ & $30.76(15)$ & $181.13(25)$ & $1.31 \mathrm{e}+3(45)$ & $1.02 \mathrm{e}+4(90)$ \\
$4 \times 4$ & & $5.55(14)$ & $13.30(20)$ & $61.52(32)$ & $399.89(72)$ & $3.01 \mathrm{e}+3(161)$ \\
$8 \times 8$ & & & $5.70(15)$ & $19.23(26)$ & $99.80(53)$ & $679.15(117)$ \\
$16 \times 16$ & & & & $6.01(16)$ & $23.24(28)$ & $128.24(59)$ \\
$32 \times 32$ & & & & & $5.86(15)$ & $24.24(28)$ \\
$64 \times 64$ & & & & & & $5.81(15)$
\end{tabular}

OAS(2) prec. with $r=1, \quad p=3, k=2$ B-splines, square domain

\begin{tabular}{c|r|r|r|r|r|r} 
& $1 / h=8$ & $1 / h=16$ & $1 / h=32$ & $1 / h=64$ & $1 / h=128$ & $1 / h=256$ \\
$N$ & $\kappa_{2}$ (it) & $\kappa_{2}$ (it) & $\kappa_{2}$ (it) & $\kappa_{2}(\mathrm{it})$ & $\kappa_{2}$ (it) & $\kappa_{2}$ (it) \\
\hline $2 \times 2$ & $4.50(9)$ & $4.64(11)$ & $11.10(14)$ & $50.12(19)$ & $323.44(31)$ & $2.43 e+3(56)$ \\
$4 \times 4$ & & $4.56(15)$ & $6.14(16)$ & $19.90(24)$ & $105.06(43)$ & $728.39(94)$ \\
$8 \times 8$ & & & $4.53(15)$ & $7.67(19)$ & $30.23(34)$ & $173.98(71)$ \\
$16 \times 16$ & & & & $4.58(15)$ & $8.78(20)$ & $37.40(37)$ \\
$32 \times 32$ & & & & & $4.59(15)$ & $9.02(20)$ \\
$64 \times 64$ & & & & & & $4.60(15)$
\end{tabular}

TABLE 5.4

$2 D$ OAS(2) scalability and optimality test on the quarter-ring domain: condition number of the preconditioned operator $\left(\kappa_{2}\right)$ and PCG iteration counts (it) in brackets as a function of the number of subdomains $N$ and mesh size $1 / h . p=3, k=2$ NURBS.

OAS(2) prec. with $r=0, p=3, k=2$ NURBS, quarter-ring domain

\begin{tabular}{c|r|r|r|r|r|r} 
& $1 / h=8$ & $1 / h=16$ & $1 / h=32$ & $1 / h=64$ & $1 / h=128$ & $1 / h=256$ \\
$N$ & $\kappa_{2}$ (it) & $\kappa_{2}(\mathrm{it})$ & $\kappa_{2}(\mathrm{it})$ & $\kappa_{2}(\mathrm{it})$ & $\kappa_{2}(\mathrm{it})$ & $\kappa_{2}(\mathrm{it})$ \\
\hline $2 \times 2$ & $8.06(13)$ & $30.78(18)$ & $185.31(34)$ & $1.37 \mathrm{e}+3(65)$ & $1.07 \mathrm{e}+4(138)$ & $8.56 \mathrm{e}+4(294)$ \\
$4 \times 4$ & & $18.52(24)$ & $99.55(47)$ & $703.41(114)$ & $5.45 \mathrm{e}+3(294)$ & $4.34 \mathrm{e}+4(760)$ \\
$8 \times 8$ & & & $41.61(39)$ & $260.55(89)$ & $1.96 \mathrm{e}+3(231)$ & $1.55 \mathrm{e}+4(660)$ \\
$16 \times 16$ & & & & $81.08(55)$ & $538.31(137)$ & $4.13 \mathrm{e}+3(387)$ \\
$32 \times 32$ & & & & & $126.16(73)$ & $863.56(192)$ \\
$64 \times 64$ & & & & & & $131.40(75)$
\end{tabular}

OAS(2) prec. with $r=1, \quad p=3, k=2$ NURBS, quarter-ring domain

\begin{tabular}{c|r|r|r|r|r|r} 
& $1 / h=8$ & $1 / h=16$ & $1 / h=32$ & $1 / h=64$ & $1 / h=128$ & $1 / h=256$ \\
$N$ & $\kappa_{2}$ (it) & $\kappa_{2}$ (it) & $\kappa_{2}$ (it) & $\kappa_{2}$ (it) & $\kappa_{2}$ (it) & $\kappa_{2}$ (it) \\
\hline $2 \times 2$ & $5.11(11)$ & $10.90(15)$ & $50.08(23)$ & $331.40(44)$ & $2.53 \mathrm{e}+3(85)$ & $2.01 \mathrm{e}+4(180)$ \\
$4 \times 4$ & & $8.02(18)$ & $28.51(30)$ & $174.47(62)$ & $1.29 \mathrm{e}+3(155)$ & $1.02 \mathrm{e}+4(407)$ \\
$8 \times 8$ & & & $15.81(26)$ & $67.93(49)$ & $469.36(121)$ & $3.63 \mathrm{e}+3(333)$ \\
$16 \times 16$ & & & & $30.15(36)$ & $135.93(76)$ & $981.02(197)$ \\
$32 \times 32$ & & & & & $46.00(46)$ & $215.36(98)$ \\
$64 \times 64$ & & & & & & $47.84(47)$
\end{tabular}

First, we remark that the unpreconditioned problem is extremely ill-conditioned, reaching, for $p=12$, values around $O\left(10^{10}\right)$ for $k=p-1$ and $O\left(10^{11}\right)$ for $k=1$. In spite of this, the OAS preconditioner performs quite well in the case of generous overlap $r=p$ since the condition numbers decrease (unexpectedly) below 5 and the iteration counts stabilize around 18 . When the overlap is minimal, the performance suffers but still seems to be independent of $p$ for $k=1$, while it becomes quite irregular for $k=p-1$, showing an initial decrease of both $\kappa_{2}$ 


\section{ETNA}

Kent State University and

Johann Radon Institute (RICAM)

TABLE 5.5

$2 D O A S(2)$ scalability and optimality test on the unit square domain: condition number of the preconditioned operator $\left(\kappa_{2}\right)$ and PCG iteration counts (it) in brackets as a function of the number of subdomains $N$ and mesh size $1 / h . p=3, k=1$ B-splines.

$\mathrm{OAS}(2)$ prec. with $r=0, p=3, k=1 \mathrm{~B}$-splines, square domain

\begin{tabular}{c|r|r|r|r|r|r} 
& $1 / h=8$ & $1 / h=16$ & $1 / h=32$ & $1 / h=64$ & $1 / h=128$ & $1 / h=256$ \\
$N$ & $\kappa_{2}$ (it) & $\kappa_{2}$ (it) & $\kappa_{2}$ (it) & $\kappa_{2}$ (it) & $\kappa_{2}$ (it) & $\kappa_{2}$ (it) \\
\hline $2 \times 2$ & $6.01(10)$ & $19.56(13)$ & $106.37(21)$ & $747.17(39)$ & $5.77 \mathrm{e}+3(76)$ & $4.57 \mathrm{e}+4(164)$ \\
$4 \times 4$ & & $6.73(17)$ & $20.19(26)$ & $105.69(52)$ & $732.71(120)$ & $5.64 \mathrm{e}+3(281)$ \\
$8 \times 8$ & & & $7.26(19)$ & $21.53(32)$ & $108.47(69)$ & $744.56(159)$ \\
$16 \times 16$ & & & & $7.35(19)$ & $22.81(31)$ & $116.89(73)$ \\
$32 \times 32$ & & & & & $7.34(19)$ & $23.17(33)$ \\
$64 \times 64$ & & & & & & $7.31(19)$
\end{tabular}

OAS(2) prec. with $r=1, p=3, k=1$ B-splines, square domain

\begin{tabular}{c|r|r|r|r|r|r} 
& $1 / h=8$ & $1 / h=16$ & $1 / h=32$ & $1 / h=64$ & $1 / h=128$ & $1 / h=256$ \\
$N$ & $\kappa_{2}(\mathrm{it})$ & $\kappa_{2}(\mathrm{it})$ & $\kappa_{2}(\mathrm{it})$ & $\kappa_{2}(\mathrm{it})$ & $\kappa_{2}(\mathrm{it})$ & $\kappa_{2}(\mathrm{it})$ \\
\hline $2 \times 2$ & $4.17(10)$ & $5.98(12)$ & $19.49(16)$ & $106.01(24)$ & $746.44(42)$ & $5.77 \mathrm{e}+3(79)$ \\
$4 \times 4$ & & $4.68(16)$ & $6.75(19)$ & $20.17(28)$ & $105.59(54)$ & $732.22(125)$ \\
$8 \times 8$ & & & $4.81(16)$ & $7.30(20)$ & $21.56(35)$ & $108.44(72)$ \\
$16 \times 16$ & & & & $4.81(16)$ & $7.40(21)$ & $22.86(34)$ \\
$32 \times 32$ & & & & & $4.81(16)$ & $7.40(21)$ \\
$64 \times 64$ & & & & & & $4.80(16)$
\end{tabular}

TABLE 5.6

$2 D O A S(2)$ scalability and optimality test on the quarter-ring domain: condition number of the preconditioned operator $\left(\kappa_{2}\right)$ and PCG iteration counts (it) in brackets as a function of the number of subdomains $N$ and mesh size $1 / h . p=3, k=1$ NURBS.

\begin{tabular}{|c|c|c|c|c|c|c|}
\hline$N$ & $\begin{array}{c}1 / h=8 \\
\kappa_{2} \text { (it) }\end{array}$ & $\begin{array}{r}1 / h=16 \\
\kappa_{2} \text { (it) }\end{array}$ & $\begin{array}{r}1 / h=32 \\
\kappa_{2} \text { (it) }\end{array}$ & $\begin{array}{r}1 / h=64 \\
\kappa_{2} \text { (it) }\end{array}$ & $\begin{array}{r}1 / h=128 \\
\kappa_{2} \quad \text { (it) }\end{array}$ & $\begin{array}{r}1 / h=256 \\
\kappa_{2} \quad(\mathrm{it})\end{array}$ \\
\hline $2 \times 2$ & $20.16(17)$ & $108.59(29)$ & $773.26(57)$ & $6.03 e+3(116)$ & $4.81 \mathrm{e}+4(247)$ & $3.85 e+5(577)$ \\
\hline $4 \times 4$ & & 29.07 (34) & $171.07(71)$ & $1.27 \mathrm{e}+3(183)$ & $1.00 \mathrm{e}+4(491)$ & $8.02 \mathrm{e}+4(1347)$ \\
\hline $8 \times 8$ & & & $36.43(43)$ & 224.68 (107) & $1.70 \mathrm{e}+3(288)$ & $1.35 e+4(824)$ \\
\hline $16 \times 16$ & & & & $42.32(50)$ & 279.17 (133) & $2.17 e+3(371)$ \\
\hline $32 \times 32$ & & & & & $46.75(53)$ & $325.98(142)$ \\
\hline $64 \times 64$ & & & & & & $50.40(56)$ \\
\hline
\end{tabular}

OAS(2) prec. with $r=1, \quad p=3, k=1 \mathrm{~B}$-splines, quarter-ring domain

\begin{tabular}{c|r|r|r|r|r|r} 
& $1 / h=8$ & $1 / h=16$ & $1 / h=32$ & $1 / h=64$ & $1 / h=128$ & $1 / h=256$ \\
$N$ & $\kappa_{2}$ (it) & $\kappa_{2}$ (it) & $\kappa_{2}$ (it) & $\kappa_{2}$ (it) & $\kappa_{2}$ (it) & $\kappa_{2}$ (it) \\
\hline $2 \times 2$ & $6.78(13)$ & $20.22(19)$ & $108.33(30)$ & $771.55(60)$ & $6.02 \mathrm{e}+3(121)$ & $4.80 \mathrm{e}+4(254)$ \\
$4 \times 4$ & & $8.32(21)$ & $29.22(36)$ & $170.64(74)$ & $1.26 \mathrm{e}+3(194)$ & $1.00 \mathrm{e}+4(529)$ \\
$8 \times 8$ & & & $9.68(23)$ & $36.51(45)$ & $224.00(111)$ & $1.69 \mathrm{e}+3(305)$ \\
$16 \times 16$ & & & & $10.83(26)$ & $42.62(53)$ & $277.79(140)$ \\
$32 \times 32$ & & & & & $11.66(27)$ & $47.08(58)$ \\
$64 \times 64$ & & & & & & $12.23(28)$
\end{tabular}

and iteration counts followed by a strong increase with $p$, particularly for odd values.

5.3. 3D tests: OAS(2) weak scalability. Table 5.8 displays the results of a $3 \mathrm{D}$ weak scalability test on a cubic domain (left columns) and a thick quarter-ring domain (right columns) for two levels of overlap $r=0,1$. We fix $H / h=4$ and $p=2, k=1$ (top table) or $p=3, k=2$ (bottom table), increasing the number of subdomains $N$. The results show 
ETNA

Kent State University and

Johann Radon Institute (RICAM)
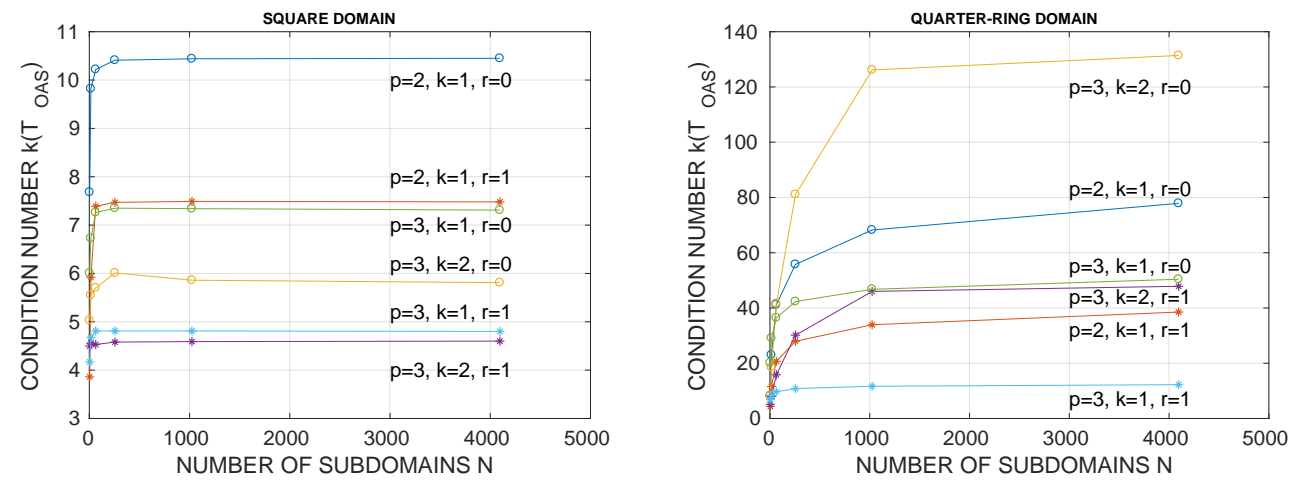

FIG. 5.2. Scalability plots of $\kappa_{2}\left(T_{O A S}\right)$ for increasing $N$ from the diagonals $(H / h=4)$ of Tables 5.1-5.6. Square domain (left) and quarter-ring domain (right).
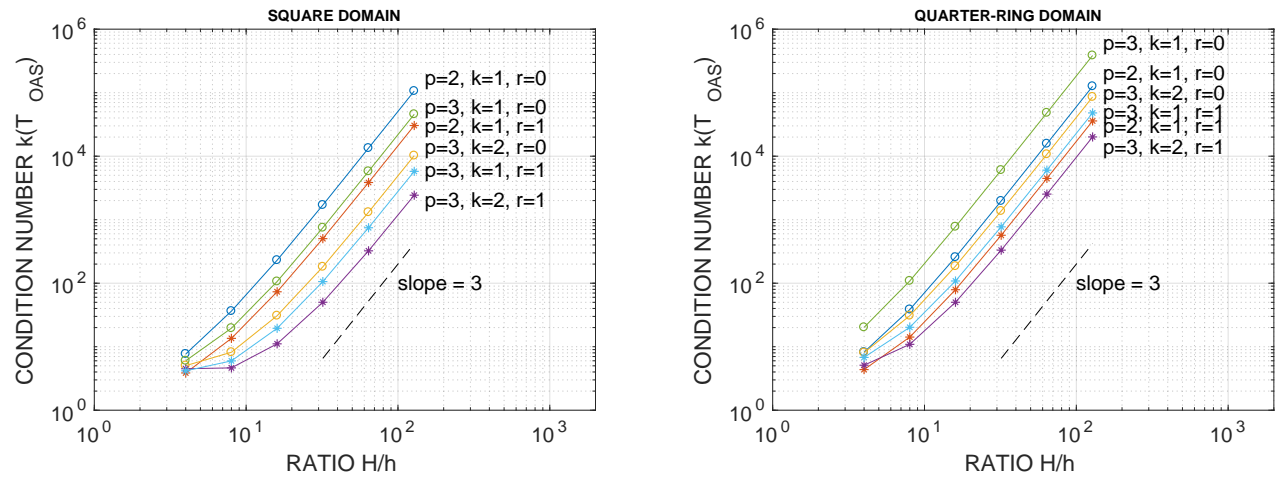

FIG. 5.3. Quasi-optimality plots of $\kappa_{2}\left(T_{O A S}\right)$ for increasing $H / h$ from the the first rows $(N=2 \times 2)$ of Tables 5.1-5.6. Square domain (left) and quarter-ring domain (right).
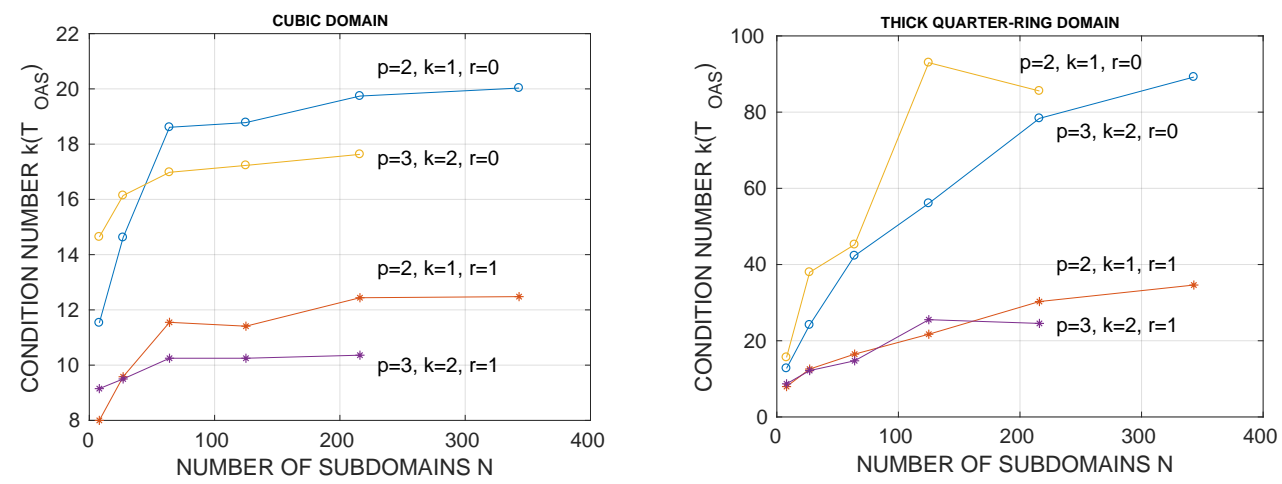

FIG. 5.4. Scalability plots of $\kappa_{2}\left(T_{O A S}\right)$ for increasing $N$ from Table 5.8. Cubic domain (left) and thick quarter-ring domain (right). 


\section{ETNA}

Kent State University and

Johann Radon Institute (RICAM)

TABLE 5.7

$O A S(2)$ dependence on $p$ and $k$ on the quarter-ring domain: condition number of the preconditioned operator $\left(\kappa_{2}\right)$ and PCG iteration counts (it) in brackets as a function of polynomial degree p for maximal regularity $k=p-1$ (left) and minimal regularity $k=1$ (right), with different levels of overlap from symmetric minimal $(r=0)$ to symmetric generous $(r=p)$. Fixed $1 / h=32, N=2 \times 2, H / h=16$.

\begin{tabular}{r|rrr|rrr} 
& \multicolumn{4}{|c|}{$k=p-1$} & \multicolumn{4}{c}{$k=1$} \\
\multicolumn{1}{c|}{ no prec. } & \multicolumn{2}{|c|}{ OAS(2) prec. } & no prec. & \multicolumn{2}{c}{ OAS(2) prec. } \\
& & \multicolumn{2}{c}{$r=0$} & $r=p$ & & \multicolumn{2}{r}{$r=0$} & $r=p$ \\
& & $\kappa_{2}$ (it) & $\kappa_{2}$ (it) & & $\kappa_{2}$ (it) & $\kappa_{2}$ (it) \\
\hline 2 & $5.6 \mathrm{e}+4$ & $256.35(33)$ & - & $5.6 \mathrm{e}+4$ & $256.35(33)$ & $36.51(19)$ \\
3 & $2.2 \mathrm{e}+4$ & $185.31(34)$ & $50.08(23)$ & $4.1 \mathrm{e}+4$ & $773.26(57)$ & $20.24(20)$ \\
4 & $3.2 \mathrm{e}+4$ & $72.75(26)$ & $29.13(21)$ & $1.5 \mathrm{e}+6$ & $403.61(54)$ & $8.52(19)$ \\
5 & $4.9 \mathrm{e}+4$ & $50.64(26)$ & $9.84(17)$ & $4.2 \mathrm{e}+6$ & $288.73(57)$ & $5.85(18)$ \\
6 & $7.8 \mathrm{e}+4$ & $23.25(22)$ & $7.74(17)$ & $9.9 \mathrm{e}+6$ & $242.31(62)$ & $5.02(19)$ \\
7 & $3.1 \mathrm{e}+5$ & $33.02(27)$ & $5.41(16)$ & $1.9 \mathrm{e}+7$ & $222.70(63)$ & $4.95(18)$ \\
8 & $3.4 \mathrm{e}+6$ & $11.74(19)$ & $5.06(15)$ & $4.5 \mathrm{e}+7$ & $215.00(65)$ & $4.91(18)$ \\
9 & $3.5 \mathrm{e}+7$ & $133.50(45)$ & $5.96(17)$ & $1.8 \mathrm{e}+8$ & $213.30(65)$ & $4.94(18)$ \\
10 & $3.6 \mathrm{e}+8$ & $12.30(22)$ & $4.86(15)$ & $2.0 \mathrm{e}+9$ & $214.81(58)$ & $4.95(18)$ \\
11 & $3.5 \mathrm{e}+9$ & $802.55(76)$ & $6.79(18)$ & $2.6 \mathrm{e}+10$ & $195.21(64)$ & $4.94(18)$ \\
12 & $3.6 \mathrm{e}+10$ & $75.88(37)$ & $4.96(16)$ & $4.1 \mathrm{e}+11$ & $222.12(67)$ & $4.92(18)$
\end{tabular}

TABLE 5.8

$3 D$ OAS(2) weak scalability test on a cubic domain (left) and thick quarter-ring domain (right): condition numbers of the preconditioned operator $\left(\kappa_{2}\right)$ and PCG iteration counts (it) as a function of the number of subdomains $N$, for overlap $r=0$ and $r=1$. Fixed $H / h=4$ and $p=2, k=1$ (top table), $p=3, k=2$ (bottom table).

\begin{tabular}{|c|c|c|c|c|c|c|c|c|c|}
\hline & \multirow[b]{3}{*}{$N$} & \multicolumn{4}{|c|}{ cubic domain } & \multicolumn{4}{|c|}{ thick quarter-ring } \\
\hline & & \multicolumn{2}{|c|}{$r=0$} & \multicolumn{2}{|c|}{$r=1$} & \multicolumn{2}{|c|}{$r=0$} & \multicolumn{2}{|c|}{$r=1$} \\
\hline & & $\kappa_{2}$ & it & $\kappa_{2}$ & it & $\kappa_{2}$ & it & $\kappa_{2}$ & it \\
\hline \multirow{6}{*}{$\begin{array}{l}p=2 \\
k=1\end{array}$} & $\times 2 \times 2$ & 1.53 & 13 & & 11 & 75 & 17 & 97 & 13 \\
\hline & & & 18 & & 18 & 14 & 24 & & 22 \\
\hline & & & 23 & & 21 & .30 & 31 & .49 & 21 \\
\hline & 5 & 78 & 26 & & 23 & .06 & 38 & 1.71 & 29 \\
\hline & $6 \times 6$ & 19.74 & 29 & & 25 & 78.36 & 46 & 30.29 & 33 \\
\hline & $7 \times 7 \times 7$ & 20.03 & 30 & 12.48 & 25 & 89.21 & 50 & 34.62 & 36 \\
\hline \multirow{5}{*}{$\begin{array}{l}p=3 \\
k=2\end{array}$} & $2 \times 2 \times 2$ & 14.64 & 16 & 9.15 & 15 & 15.63 & 19 & 8.70 & 14 \\
\hline & $3 \times 3 \times 3$ & 16.14 & 19 & 9.50 & 21 & 37.97 & 29 & 12.17 & 23 \\
\hline & $4 \times 4 \times 4$ & 16.98 & 24 & 10.25 & 23 & 45.23 & 35 & 14.74 & 25 \\
\hline & $5 \times 5 \times 5$ & 17.23 & 23 & 10.25 & 23 & 92.98 & 46 & 25.53 & 31 \\
\hline & $6 \times 6 \times 6$ & 17.63 & 25 & 10.36 & 23 & 85.55 & 50 & 24.55 & 30 \\
\hline
\end{tabular}

the scalability of our OAS biharmonic preconditioner since the condition numbers $\kappa_{2}\left(T_{O A S}\right)$ and iteration counts are clearly bounded from above as $N$ increases; see also Figure 5.4. For the thick quarter-ring, the performance of the OAS preconditioner suffers from a worse conditioning due to the domain deformation, but the results still seem to approach the scalable regime (we could not further increase the number of subdomains due to memory constraints). For both domains, the preconditioner performance improves when the overlap size is increased.

6. Conclusions. We have constructed a two-level additive Schwarz preconditioner for conforming isogeometric discretizations of the biharmonic equation in two and three spatial dimensions. We have proved that the resulting algorithm is scalable and quasi-optimal. 
Extensive numerical tests have validated the theoretical results and have investigated the behavior of the preconditioner with respect to the polynomial degree and global regularity of the spline functions.

A limitation of the present paper is that we have considered subdomain partitions constructed via only the reference patch. Future works should be devoted to the extension of the proposed additive Schwarz method to multipatch isogeometric discretizations of the biharmonic problem and to other $H^{2}$-problems such as Kirchhoff-Love plates and shells.

\section{REFERENCES}

[1] H. AdiBi AND J. Es'HAGHI, Numerical solution for biharmonic equation using multilevel radial basis functions and domain decomposition methods, Appl. Math. Comput., 186 (2007), pp. 246-255.

[2] A. Avundainayagam And C. VAni, A domain decomposition method for biharmonic equation, Comput. Math. Appl., 40 (2000), pp. 865-876.

[3] Y. Bazilevs, L. Beirão da Veiga, J. A. Cottrell, T. J. R. Hughes, and G. Sangalli, Isogeometric analysis: approximation, stability and error estimates for h-refined meshes, Math. Models Methods Appl. Sci., 16 (2006), pp. 1-60.

[4] L. Beirão da Veiga, A. Buffa, D. Cho, And G. SAngalli, IsoGeometric Analysis using T-splines on two-patch geometries, Comput. Meth. Appl. Mech. Engrg., 200 (2011), pp. 1787-1803.

[5] L. Beirẽo Da Veiga, A. Buffa, J. Rivas, AND G. SANGalli, Some estimates for $h$-p- $k$-refinement in isogeometric analysis, Numer. Math., 118 (2011), pp. 271-305.

[6] L. Beirão DA Veiga, D. Cho, L. F. PaVARINo, AND S. SCACChI, Overlapping Schwarz methods for isogeometric analysis, SIAM J. Numer. Anal., 50 (2012), pp. 1394-1416.

[7] — BDDC preconditioners for isogeometric analysis, Math. Models Methods Appl. Sci., 23 (2013), pp. 1099-1142.

[8] —, Isogeometric Schwarz preconditioners for linear elasticity systems, Comput. Meth. Appl. Mech. Engrg., 253 (2013), pp. 439-454,.

[9] - Overlapping Schwarz preconditioners for isogeometric collocation methods, Comput. Meth. Appl. Mech. Engrg., 278 (2014), pp. 239-253.

[10] L. BeIRÃo DA VeIga, D. Cho, AND G. SANGalli, Anisotropic NURBS approximation in isogeometric Analysis, Comput. Meth. Appl. Mech. Engrg., 209/212 (2012), pp. 1-11.

[11] L. Beir Ão DA VeIga, L. F. PAVARINo, S. SCACChI, O. B. WidLund, AND S. ZAMPini, Isogeometric BDDC preconditioners with deluxe scaling, SIAM J. Sci. Comp., 36 (2014), pp. A1118-A1139.

[12] — Adaptive selection of primal constraints for isogeometric BDDC deluxe preconditioners, SIAM J. Sci. Comp., 39 (2017), pp. A281-A302.

[13] B. Bialecki, X.-C. CAI, M. DRYja, AND G. FAIRWEATHER, An additive Schwarz algorithm for piecewise Hermite bicubic orthogonal spline collocation, in Domain Decomposition Methods in Science and Engineering, A. Quarteroni, J. Périaux, and Y. A. Kuznetsov, eds., Contemp. Math., 157, American Mathematical Society, Providence, 1994, pp. 237-244.

[14] B. BIALECKI AND M. DRYJA, Multilevel additive and multiplicative methods for orthogonal spline collocation problems, Numer. Math., 77 (1997), pp. 35-58.

[15] S. Brenner And R. Scott, The Mathematical Theory of Finite Element Methods, Springer, New York, 2008.

[16] S. C. BRENNER AND K. WANG, Two-level additive Schwarz preconditioners for $C^{0}$ interior penalty methods, Numer. Math., 102 (2005), pp. 231-255.

[17] _ An iterative substructuring algorithm for a CO interior penalty method, Electron. Trans. Numer. Anal., 39 (2012), pp 313-332.

http://etna.ricam.oeaw.ac.at/vol.39.2012/pp313-332.dir/pp313-332.pdf

[18] A. Buffa, H. Harbrecht, A. Kunoth, And G. SAngalli, BPX-preconditioning for isogeometric analysis, Comput. Meth. Appl. Mech. Engrg., 265 (2013), pp. 63-70.

[19] P. G. CIARLET AND P.-A. RAVIART, A mixed finite element method for the biharmonic equation, in Mathematical Aspects of Finite Elements in Partial Differential Equations, Proc. Sympos., Math. Res. Center Univ. Wisconsin 1974, C. de Boor, ed., Publication No. 33. of Math. Res. Center Univ. of Wisconsin-Madison, Academic Press, New York, 1974, pp. 125-145.

[20] P. Costantini, C. Manni, F. Pelosi, And M. L. Sampoli, Quasi-interpolation in isogeometric analysis based on generalized B-splines, Comput. Aided Geom. Design, 27 (2010), pp. 656-668.

[21] J. A. Cottrell, T. J. R. Hughes, AND Y. BAZILEVs, Isogeometric Analysis. Towards integration of CAD and FEA, Wiley, Chichester, 2009.

[22] C. DE FALCO, A. REALI, AND R. VAZQUEZ, GeoPDEs: a research tool for isogeometric analysis of PDEs, Adv. Engrg. Softw., 42 (2011), pp. 1020-1034. 
[23] M. Donatelli, C. Garoni, C. Manni, S. Serra-Capizzano, and H. Speleers Robust and optimal multi-iterative techniques for IgA Galerkin linear systems, Comput. Meth. Appl. Mech. Engrg., 284 (2015), pp. 230-264.

[24] M. DRYJA AND O. B. WidLund, Domain decomposition methods with small overlap, SIAM J. Sci. Comput., 15 (1994), pp. 604-620.

[25] C. FARHAT AND J. MANDEL, The two-level FETI method for static and dynamic plate problems. I. An optimal iterative solver for biharmonic systems, Comput. Meth. Appl. Mech. Engrg., 155 (1998), pp. 129-151.

[26] G. E. FARIN, NURBS Curves and Surfaces: From Projective Geometry to Practical Use, A.K. Peters, Wellesley, 1995.

[27] X. FENG AND O. A. KARAKASHIAN, Two-level non-overlapping Schwarz preconditioners for a discontinuous Galerkin approximation of the biharmonic equation, J. Sci. Comput., 22/23 (2005), pp. 289-314.

[28] P. F. FISCHER AND J.W. LOTTES, Hybrid Schwarz-multigrid methods for the spectral element method: extensions to Navier-Stokes, Domain Decomposition Methods in Science and Engineering, R. Kornhuber, R. Hoppe, J. Périaux, Ol. Pironneau, O. Widlund, and J. Xu, eds., Lect. Notes Comput. Sci. Eng., 40, Springer, Berlin, 2005, pp. 35-49.

[29] K. Gahalaut, J. Kraus, and S. Tomar, Multigrid methods for isogeometric discretization, Comput. Meth. Appl. Mech. Engrg., 253 (2013), pp. 413-425.

[30] T. J. R. Hughes, J. A. CotTRELl, AND Y. BAZILEvs, Isogeometric analysis: CAD, finite elements, NURBS, exact geometry, and mesh refinement, Comput. Meth. Appl. Mech. Engrg., 194 (2005), pp. 4135-4195.

[31] M. KAPL, F. BUCHEGGER, M. BERCOVIER, AND B. JÜTTLER, Isogeometric analysis with geometrically continuous functions on planar multi-patch geometries, Comput. Meth. Appl. Mech. Engrg., 316 (2017), pp. 209-234.

[32] S. K. Kleiss, C. Pechstein, B. JÜtTLER, And S. Tomar, IETI-isogeometric tearing and interconnecting, Comput. Meth. Appl. Mech. Engrg., 247/248 (2012), pp. 201-215.

[33] K-A. Mardal, B. F. NIELSEn, AND M. NoRdaAs, Robust preconditioners for PDE-constrained optimization with limited observations BIT, 57 (2007), pp. 405-431.

[34] P. MonK, A mixed finite element method for the biharmonic equation, SIAM J. Numer. Anal., 24 (1987), pp. 737-749.

[35] S. MOORE, Discontinuous Galerkin isogeometric analysis for the biharmonic equation, Preprint on arXiv, https://arxiv.org/abs/1703.02726v1, 2017.

[36] L. F. PAVARINO AND S. SCACCHI, Isogeometric block FETI-DP preconditioners for the Stokes and mixed linear elasticity systems, Comput. Meth. Appl. Mech. Engrg., 310 (2016), pp. 694-710.

[37] L.L. SchumaKer, Spline Functions: Basic Theory, 3rd ed, Cambridge University Press, Cambridge, 2007.

[38] B. F. Smith, P. E. BJøRStad, AND W. Gropp, Domain Decomposition: Parallel Multilevel Methods for Elliptic Partial Differential Equations, Cambridge University Press, Cambridge, 1996.

[39] J. SOGN AND W. ZULEHNER, Robust preconditioners for PDE-constrained optimization with limited observations using isogeometric discretization, Tech. Report G+S Report No. 34, University Linz, 2015.

[40] A. TAgliabue, L. DedĖ, AND A. Quarteroni, Isogeometric Analysis and error estimates for high order partial differential equations in fluid dynamics, Comput. \& Fluids, 102 (2014), pp. 277-303.

[41] A. Toselli AND O. Widlund, Domain Decomposition Methods-Algorithms and Theory, Springer, Berlin, 2005.

[42] X. Zhang, Multilevel Schwarz methods for the biharmonic Dirichlet problem, SIAM J. Sci. Comput., 15 (1994), pp. 621-644.

[43] - Two-level Schwarz methods for the biharmonic problem discretized conforming $C^{1}$ elements, SIAM J. Numer. Anal, 33 (1996), pp. 555-570. 